\title{
Cortical Neuron Response Properties Are Related to Lesion Extent and Behavioral Recovery after Sensory Loss from Spinal Cord Injury in Monkeys
}

\author{
Hui-Xin Qi, ${ }^{\star}$ Jamie L. Reed, ${ }^{*}$ Omar A. Gharbawie, Mark J. Burish, and Jon H. Kaas \\ Department of Psychology, Vanderbilt University, Nashville, Tennessee 37240
}

\begin{abstract}
Lesions of the dorsal columns at a mid-cervical level render the hand representation of the contralateral primary somatosensory cortex (area 3b) unresponsive. Over weeks of recovery, most of this cortex becomes responsive to touch on the hand. Determining functional properties of neurons within the hand representation is critical to understanding the neural basis of this adaptive plasticity. Here, we recorded neural activity across the hand representation of area $3 \mathrm{~b}$ with a 100 -electrode array and compared results from owl monkeys and squirrel monkeys 5-10 weeks after lesions with controls. Even after extensive lesions, performance on reach-to-grasp tasks returned to prelesion levels, and hand touches activated territories mainly within expected cortical locations. However, some digit representations were abnormal, such that receptive fields of presumably reactivated neurons were larger and more often involved discontinuous parts of the hand compared with controls. Hand stimulation evoked similar neuronal firing rates in lesion and control monkeys. By assessing the same monkeys with multiple measures, we determined that properties of neurons in area $3 \mathrm{~b}$ were highly correlated with both the lesion severity and the impairment of hand use. We propose that the reactivation of neurons with near-normal response properties and the recovery of near-normal somatotopy likely supported the recovery of hand use. Given the near-completeness of the more extensive dorsal column lesions we studied, we suggest that alternate spinal afferents, in addition to the few spared primary axon afferents in the dorsal columns, likely have a major role in the reactivation pattern and return of function.
\end{abstract}

Key words: area 3b; dorsal column lesion; multielectrode array; primate; somatotopy; tactile

\section{Introduction}

After nearly complete lesions of sensory afferents in the dorsal column (DC) of the spinal cord, the hand representation in contralateral primary somatosensory cortex (area $3 \mathrm{~b}$ ) of monkeys becomes deactivated and hand use is impaired. Nevertheless, neuronal responses to touch on the hand return over a few postlesion weeks, and hand use recovers (Jain et al., 1997, 1998, 2008; Qi et al., 2011, 2013; Chen et al., 2012). A critical finding of these studies is that the sparing of even a small proportion of the dorsal column afferents from the hand promotes the extent of the reactivation of cortex and the recovery of hand use. However, little is

Received Nov. 25, 2013; revised Jan. 29, 2014; accepted Feb. 18, 2014.

Author contributions: H.-X.Q., J.L.R., and J.H.K. designed research; H.-X.Q., J.L.R., 0.A.G., and M.J.B. performed research; H.-X.Q. and J.L.R. analyzed data; H.-X.Q., J.L.R., and J.H.K. wrote the paper.

This work was supported by National Institutes of Health Grant NS16446 to J.H.K., Christopher and Dana Reeve Foundation to J.H.K. and J.L.R., National Institutes of Health Grant NS067017 to H.-X.Q., National Institutes of Health Grant NS053231 to J.L.R., National Institutes of Health Grant K99 NS079471-01, and the Canadian Institutes of Health Research postdoctoral fellowship to 0.A.G. We thank Laura Trice for technical support; Drs. Melanie R. Bernard, A. B. Bonds, Corrie Camalier, and Zhiyi Zhou for assistance in data collection; and Dr. Pierre Pouget for analytical tool development.

The authors declare no competing financial interests.

${ }^{*}$ H.-X.Q. and J.L.R. contributed equally to this work.

Correspondence should be addressed to Dr. Hui-Xin Qi, Vanderbilt University Department of Psychology, 301 Wilson Hall, 111 21st Avenue South, Nashville, TN 37240. E-mail: huixin.qi@vanderbilt.edu.

M.J. Burish's present address: University of California San Francisco Medical Center, 505 Parnassus Avenue, San Francisco, CA 94143

DOI:10.1523/JNEUROSCI.4954-13.2014

Copyright $\odot 2014$ the authors $\quad 0270-6474 / 14 / 344345-19 \$ 15.00 / 0$ known about the response properties of reactivated cortical neurons. According to previous subjective measures after various sensory deprivations, reactivated cortical neurons often responded well to digit tactile stimulation (Merzenich et al., 1983a,b; Tommerdahl et al., 1996; Florence et al., 1998; DarianSmith and Brown, 2000) and therefore seemed capable of restoring cortical somatosensory network function to levels that would be useful in guiding the recovery of manual dexterity. Recently, Wang et al. (2013) found dissociation between local field potentials and spiking activity in area $3 \mathrm{~b}$ and $\mathrm{S} 2$ at high stimulus frequencies after dorsal column lesion in squirrel monkeys (SMs) using single electrode recordings. Still, many other aspects of neuron properties in reactivated cortex remain to be investigated, especially the links between the cortical activity, lesion extent, and behavioral recovery.

The present study extends this body of research by quantifying neuronal response characteristics in reactivated somatosensory cortex in relation to quantified measures of the extents of dorsal column lesions, and the recovery of hand use. From these studies, we expected that neurons in reactivated cortex would have response properties that are similar to those of neurons in monkeys without dorsal column lesions. However, we did not expect reactivated neurons to be completely normal, and the most severe abnormalities were expected in monkeys with the most complete lesions. To test our hypotheses, we simultaneously recorded single-neuron responses across the hand representations in area 


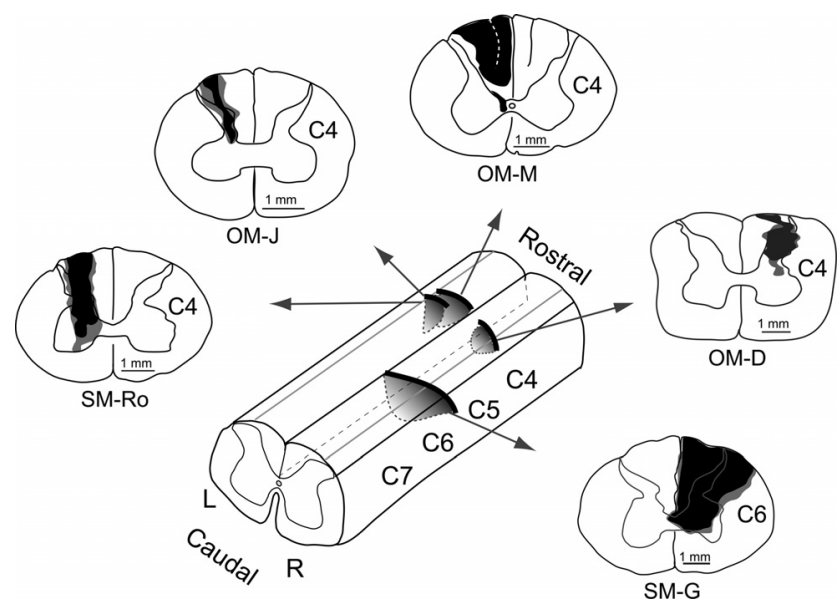

Figure 1. Schematic drawings show extents and locations of dorsal column lesions in five monkeys. A coronal view of the lesion site was reconstructed from the series of horizontally cut spinal cord sections projected into a transverse view. Black shadings represent the extent of damaged tissue; gray shadings represent surrounding affected zones. C4-C7, Spinal cord cervical segments $4-7 ; \mathrm{L}$, left; $R$, right.

3 b with a 100 electrode array, weeks after a unilateral lesion of the dorsal columns at a midcervical level in adult owl monkeys (OMs) and SMs. Both species present the advantage of having the hand representation in area $3 \mathrm{~b}$ of somatosensory cortex exposed on the brain surface for effective placement of the electrode array, and the fixed spacing of electrodes within the array assured that the distribution of neurons sampled in each case was similar. These same procedures were used to collect results from normal monkeys for comparisons to the spinal cord-lesioned monkeys. Here, we present quantitative evidence that the peak firing rates to skin indentations on the hand for reactivated neurons are indistinguishable from those in normal monkeys. Although abnormalities in receptive field (RF) sizes and locations persist, the present results suggest that neurons in reactivated cortex have sufficiently normal response properties and RF locations to contribute to the behavioral recovery process.

\section{Materials and Methods}

Eight adult New World OMs (Aotus trivirgatus, 5 females) and two adult SMs (Saimiri sciureus, 1 female) were used in the present study. Five monkeys received a unilateral lesion of the dorsal columns of the spinal cord at a C4-C6 cervical level. Five naive monkeys served as controls here and in other unrelated studies (Reed et al., 2008, 2010a, b, 2011, 2012). All experimental procedures were approved by the Vanderbilt University Animal Care and Use Committees and followed the guidelines of the National Institutes of Health Guide for the Care and Use of Laboratory Animals.

\section{Behavioral task}

The details of behavioral training and testing have been described previously (Qi et al., 2013). In brief, OMs and SMs were trained for 2 weeks before dorsal column (DC) lesion to perform a reach-to-grasp task. A modified Klüver Board $(15.2 \mathrm{~cm} \times 9.9 \mathrm{~cm})$ was mounted onto the outside of the home cage during each training sessions. The board included wells $1-4$ of decreasing diameters (range, $1.5-1.14 \mathrm{~cm}$ ) that presented increasing difficulty and increasing depths (range, $0.14-0.64 \mathrm{~cm}$ ); both factors combined into incremental levels of difficulty. Performance on the last day of training and each postlesion test day was video recorded using a Panasonic PV-GS320 (30 frames/s, 1000th of a second shutter speed). Video records were transferred onto a computer hard drive using iMovie software (Apple) and performance was analyzed frame-by-frame.

Quantitative scoring was feasible during training/testing sessions but was also confirmed from the video records. Two measures were used for
A
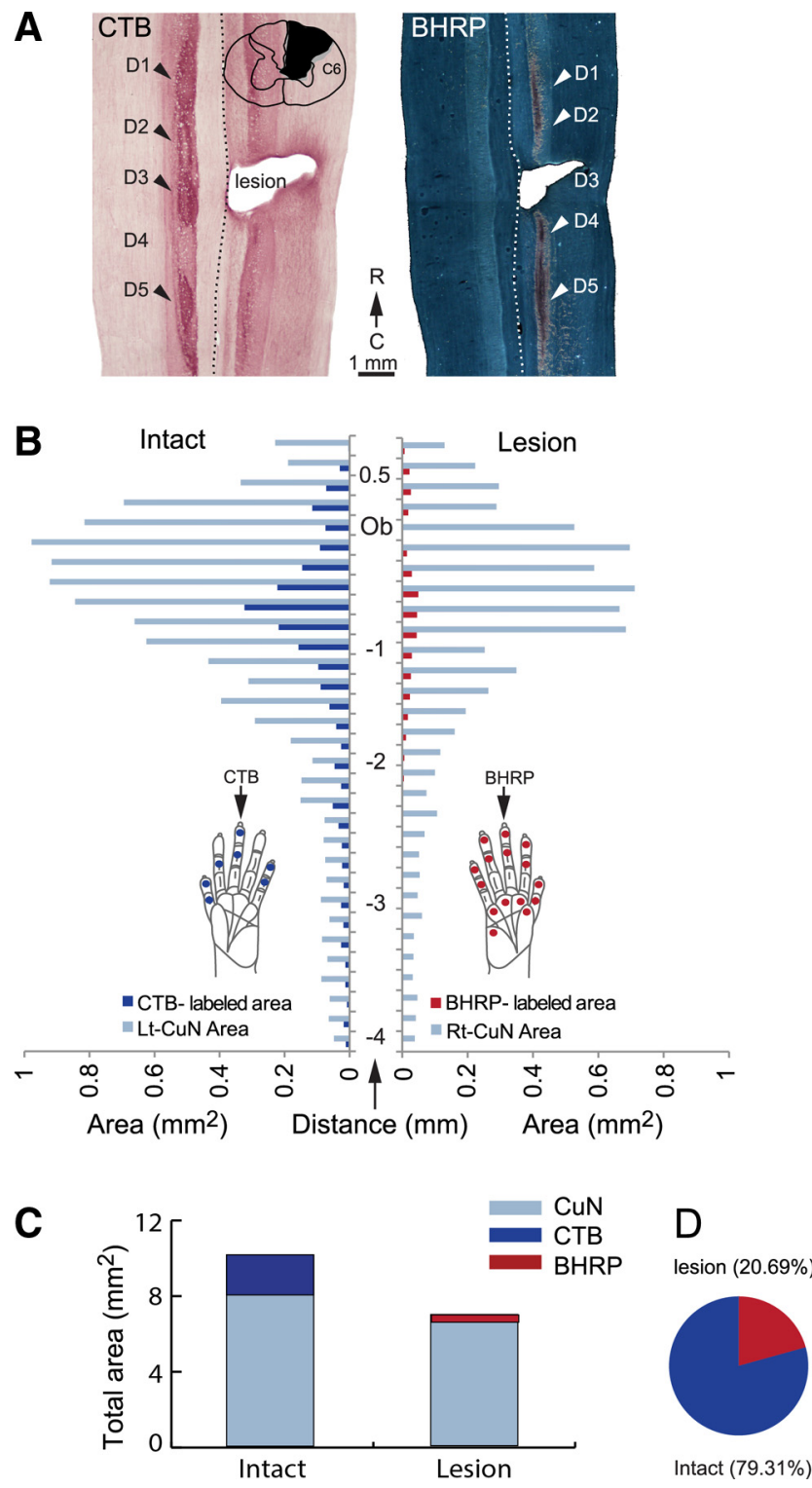

Figure 2. Estimation of lesion extent in Monkey SM-G. A, Photomicrographs from horizontally cut spinal cord sections reveal the extent and level of dorsal column lesion. Arrowheads point to CTB label (Intact) and BHRP-label (Lesion). Inset, Transverse view of spinal cord at C6 with the extent of lesion (black shading) for SM-G. B, Bar graphs contrast the areas of tracer uptake with the area of the cuneate nucleus. Distance $(\mathrm{mm})$ measured from the beginning of the obex is on the $x$-axis. Negative values show distance caudal to obex. The $y$-axis depicts the areal size for each section through the cuneate nucleus. Dots on the schematic drawings of hands indicate injection sites. C, Bar graph represents ratios of labeled area to total area of cuneate nucleus on each side. $\boldsymbol{D}$, Pie graph represents the final estimated proportion of lesion area (20.69\%) for SM-G. C4-C7, Cervical spinal cord segments 4-7; C, caudal; CuN, cuneate nucleus of the brainstem; D1-D5, digits $1-5 ; R$, rostral.

quantitative scoring. (1) Success. A successful reach is one in which the food was grasped and transferred into the mouth using the designated hand on a single trial. (2) Digit flexes per successful trial. This measure reflects the average number of digit flexes for each successfully retrieved pellet as many attempts as the monkey needed for a given pellet. If the monkey failed to retrieve a pellet for whatever reason, that trial was not included in this measure. A single flexion of the digits for a successfully retrieved pellet was considered a perfect score. Only results from the difficult wells (e.g., 3 and 4) were included in the present quantification because we already established that deeper wells are more difficult and cause performance reductions in a previous study (Qi et al., 2013). We compared prelesion scores with postlesion scores using Kruskal-Wallis 
A

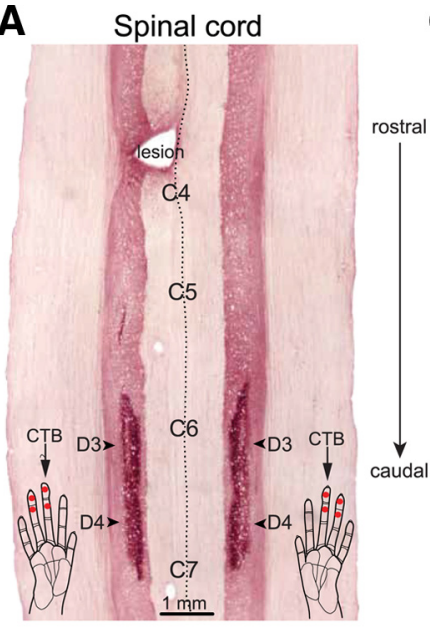

B

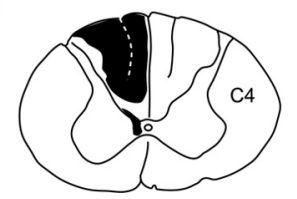

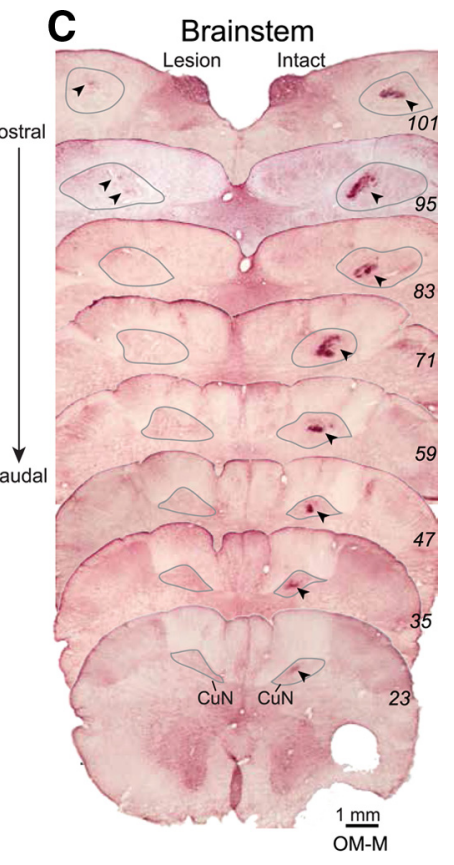

Figure 3. Terminations of peripheral afferents in the dorsal horn of the spinal cord and cuneate nucleus of the brainstem labeled by injections of CTB into the distal and middle phalanges of digits 3 and 4 of Monkey 0M-M. A, A horizontally cut CTB-immunoreacted section of the spinal cord showing the location of lesion and foci of labeled terminal fields (indicated by arrowheads) after tracer injection. Red dots on the schematic drawings of hands (inset) indicate injection sites. Dashed line indicates the midline of the spinal cord. $\boldsymbol{B}$, Drawing shows the extent of lesion (shaded in black) in the dorsal column. Lesion was reconstructed from horizontally cut immunoreacted spinal cord sections to reveal CTB tracer. Dashed line indicates the posterior fissure of the spinal cord, which separates cuneate fasciculus from gracile fasciculus. $\boldsymbol{C}$, A series of coronally cut CTB-immunoreacted sections through dorsal column nuclei of brainstem. The cuneate nucleus is outlined on both sides. There are only a few detectable foci of axon fibers on the lesion side (arrowheads). Each section is labeled with the series section number. $\mathbf{C}_{-}-\mathrm{C}$, Cervical spinal cord segments 4-7; CuN, cuneate nucleus of brainstem; D3, digit 3; D4, 4.

analysis with Dunn's test for multiple comparisons using GraphPad InStat software (GraphPad Software).

\section{DC lesion}

The details of surgical procedures have been described previously (Jain et al., 1997, 2008; Qi et al., 2011). In brief, each monkey was initially anesthetized with ketamine hydrochloride $(15 \mathrm{mg} / \mathrm{kg}$, i.m.) and then maintained in a stereotaxic head holder at a surgical level of anesthesia with $1-3 \%$ isoflurane. Heart rate, blood oxygen saturation, and levels of expired $\mathrm{CO}_{2}$ were recorded every $10 \mathrm{~min}$. Anesthesia and respiration rates were adjusted accordingly. Body temperature was monitored rectally and maintained at $37-38^{\circ} \mathrm{C}$. Under aseptic conditions, a portion of the cervical spinal cord was exposed. The DC were lesioned on one side with a fine pair of surgical scissors at either cervical level C4 (4 monkeys) or C6 of one monkey (SM-G). At each lesion site, we typically also used a microknife or a pair of fine forceps to ensure a more complete cut at that spinal cord level. Dura was replaced with Gelfilm and covered with Gelfoam. The opening was closed, and the skin sutured. Monkeys were carefully monitored until they fully recovered from anesthesia and were returned to the home cage. Monkeys received antibiotics and analgesics for 2-3 d after surgery.

\section{Tracer injection into the skin of digits}

Anatomical tracers were injected into matched digits of both hands, except in SM, Monkey SM-G (see Quantification of CTB label and Results). Our goal was to trace afferents from the skin of the hand up to their terminals in the cuneate nuclei. Comparing the density of terminals labeled between opposite sides of the cuneate nucleus allowed us to assess the extent of the dorsal column lesion and the cervical level at which it
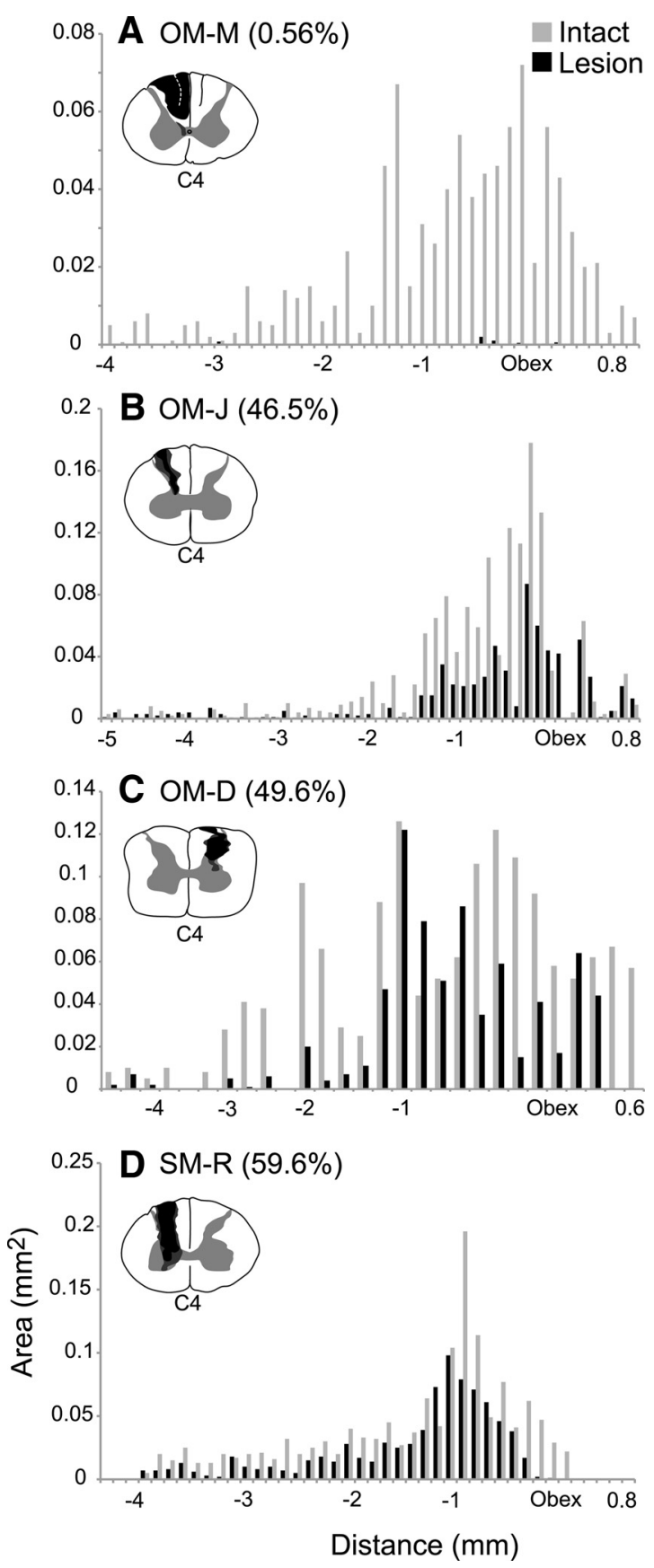

Figure 4. Bar graphs showing anatomical results from four lesion monkeys with subcutaneous CTB injections into digits of both hands. Distance $(\mathrm{mm})$ measured from the beginning of the obex is on the $x$-axis. Negative values show distance caudal to obex. The $y$-axis depicts the areal size $\left(\mathrm{mm}^{2}\right)$ of the combined foci of CTB label for each section through the cuneate nucleus of the brainstem. Values from the intact and lesion sides are compared, with the resulting proportion in parentheses, for 4 of the 5 lesion cases in $\boldsymbol{A}-\boldsymbol{D}$.

was delivered. Cholera toxin subunit B (CTB, $1 \%$ reconstitution, $5 \mu$ l per injection site, Sigma) or CTB conjugated with wheat germ agglutinin horseradish peroxide (BHRP, $0.2 \%, 5 \mu \mathrm{l}$ per injection site, List Biological) was subcutaneously injected under anesthesia (1-3\% isoflurane). Procedures followed those described previously (Florence et al., 1991; Qi and Kaas, 2006).

\section{Recording with microelectrode array and RF mapping}

Array insertion. For monkeys with dorsal column lesions, the 100 electrode array was implanted in a terminal procedure only after success scores on the reach-to-grasp task reached plateau. The array was placed 


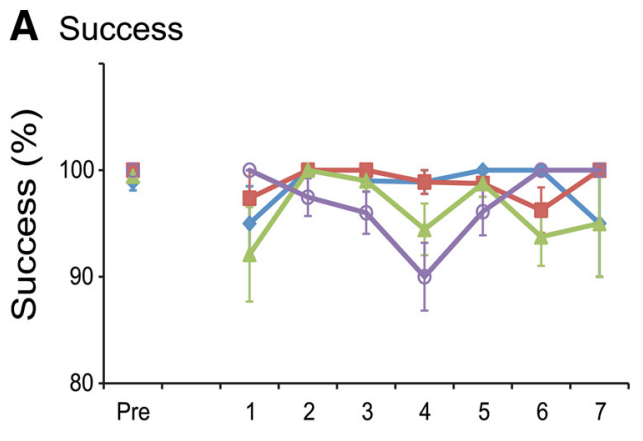

\section{B Digit flexes}

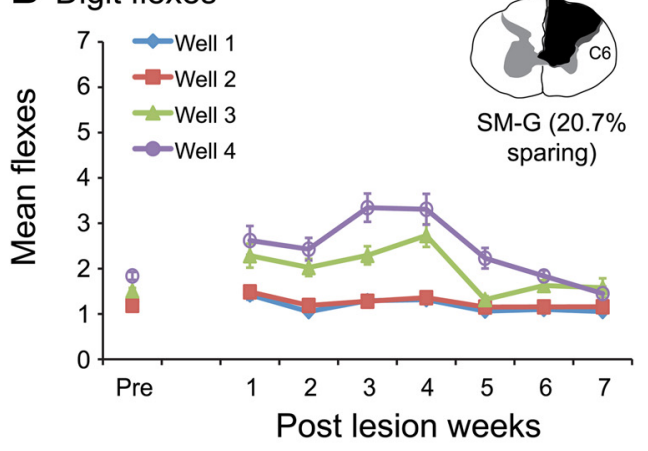

\section{Behavior and lesion correlation}

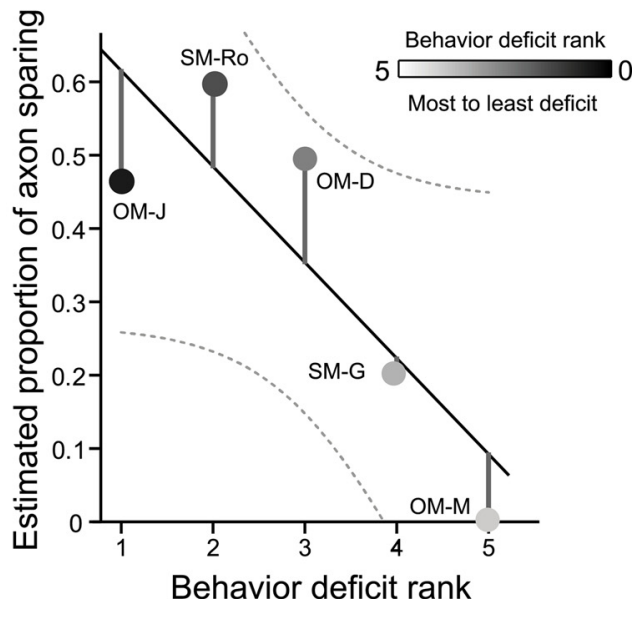

Figure 5. Example of behavioral recovery measures from Monkey SM- $G$ and summary of relationships between lesion extent and behavior deficits for all cases. $\boldsymbol{A}$, Success scores (mean \pm SEM) from Monkey SM-G performing a reach-to-grasp task. Line graphs represent postlesion changes in success score from 4 testing wells of increasing depth/difficulties, in which the well 1 (blue) is the easiest and well 4 (violet) is the most difficult. $B$, Number of digit flexes per successful trial (mean \pm SEM) from Monkey SM-G performing a reach-to-grasp task from the 4 testing wells Inset, Transverse view of spinal cord at cervical level 6 (C6) with the extent of lesion (black shading) for SM-G reconstructed from a series of horizontally cut spinal cord sections with the estimated axon sparing included for reference. C, Estimated proportion of axons spared ( $y$-axis) versus the behavior deficit rank ( $x$-axis) plotted for the 5 monkeys with dorsal column lesions shows a strong relationship between lesion extents and deficits using the impaired arm and hand. Dashed lines indicate $95 \%$ confidence intervals based on the predicted mean linear regression fit line, shown as a solid line ( $\left.y=0.75-0.13 x, R^{2}=0.731\right)$. Each lesion case $(n=5)$ is represented by a gray circle and numbered by behavioral deficit ranking from most impaired (5, light gray) to least (1, dark gray).

in somatosensory areas $3 \mathrm{~b}$ and 1 contralateral to the lesion. Surgical procedures have been fully described previously (Reed et al., 2008, 2010b). In brief, each monkey was given an initial ketamine injection (10-30 mg/kg, i.m.) for sedation followed by $2-4 \%$ halothane gas. The
Table 1. Behavior impairment rank and lesion extent ${ }^{a}$

\begin{tabular}{|c|c|c|c|c|c|}
\hline Case (survival time in weeks) & $\begin{array}{l}\text { OM-M } \\
\text { (5 weeks) }\end{array}$ & $\begin{array}{l}\text { SM-G } \\
\text { (6 weeks) }\end{array}$ & $\begin{array}{l}\text { OM-D } \\
\text { (7 weeks) }\end{array}$ & $\begin{array}{l}\text { SM-Ro } \\
\text { (8 weeks) }\end{array}$ & $\begin{array}{l}\text { OM-J } \\
\text { (10 weeks) }\end{array}$ \\
\hline Days unable to perform task & 21 & 6 & 7 & 1 & 1 \\
\hline Difference in total digit flexes & NA & 0.47 & 0.68 & 1.73 & 0.17 \\
\hline $\begin{array}{l}\text { Difference in success on last } \\
\text { testing day (\%) }\end{array}$ & NA & -5 & 6 & -1 & -5 \\
\hline Average score & NA & 3 & 2.67 & 2.33 & 2 \\
\hline Final behavior rank & 5 & 4 & 3 & 2 & 1 \\
\hline Cervical level of lesion & C4 & $\mathrm{C} 6$ & C4 & C4 & C4 \\
\hline $\begin{array}{l}\% \text { of dorsal column lesion } \\
\text { area }\end{array}$ & 81.9 & $53.8^{b}$ & 18.9 & 33.4 & 14.0 \\
\hline$\%$ of axons spared & 0.6 & 20.7 & 49.6 & 59.6 & 46.5 \\
\hline
\end{tabular}

${ }^{a}$ The five monkeys were ranked for their relative postlesion impairment in each category: 1 = least impaired, and $5=$ most impaired. The "difference" in each category is a subtraction of the mean measurements before and after DC lesion. Negative values indicate that measurements from after lesion are lower than those before lesion. " $\%$ " of dorsal column lesion area" lists cross-sectional area calculations of the reconstructed dorsal columns and lesion zones at the cervical spinal cord level.

${ }^{b}$ The value of the "\% of dorsal column lesion area" ( $93.8 \%$ ) minus $40 \%$ of estimated sparing from C 4 (20\%) and $C 5$ (20\%) because the lesion was at $(6$, which is below the level of the majority of the inputs from digits 1 and 2 ; " $\%$ of axons spared" indicates the estimate of functional axons from the hand that were spared from the lesion based on labeled axon terminals after tracer injections in the digits.

monkey was secured in a stereotaxic device, and anesthesia was maintained with propofol during surgery $(10 \mathrm{mg} / \mathrm{kg} / \mathrm{h}$, i.v. $)$ and the recordings $(0.3 \mathrm{mg} / \mathrm{kg} / \mathrm{h}$, i.v. $)$ for the duration of the experiment $(24-72 \mathrm{~h})$. Paralysis was maintained with vecuronium bromide $(0.1-0.3 \mathrm{mg} / \mathrm{kg} / \mathrm{h}$, i.v. $)$ mixed with 5\% dextrose and lactated Ringer's solution, and the monkey was artificially ventilated. Craniotomy and duratomy were performed to expose the hand representation in areas $3 \mathrm{~b}$ and 1 . The electrode array was inserted into the cortex pneumatically to a depth of $600 \mu \mathrm{m}$. Electrode tips were therefore in the expected depth of layer 3. All exposed cortex was covered with $1 \%$ agar mixed with Ringer's solution to provide stability and to prevent desiccation.

Recording. Neuronal activity was recorded with a $10 \times 10$ Utah array (Cyberkinetics Neurotechnology Systems, now Blackrock Microsystems) and the Bionics Data Acquisition System. As described in previous studies (Samonds et al., 2003; Reed et al., 2008, 2010a, b; Zhou et al., 2008), the signals on each channel were amplified by a factor of 5000 and bandpass filtered between $250 \mathrm{~Hz}$ and $7.5 \mathrm{kHz}$. The threshold for each electrode was automatically set to 3.25 SDs from the mean activity, and the waveforms were sampled at $30 \mathrm{kHz}$ for $1.5 \mathrm{~ms}$ windows.

Tactile stimulation. Tactile stimulation was applied to the skin of the hand ipsilateral-to-lesion or the skin of either hand in control cases. The hand under investigation was secured in a supine position using a Plasticine mold. A custom-designed program (Visual Basic) controlled two motor systems that implemented force- and position-feedback (300B, Aurora Scientific). The system controlled the movement of two independent probes, which were used for indenting the skin in targeted spots on the hand. The footprint of each probe was $1 \mathrm{~mm}$ in diameter. Stimuli consisted of pulses that indented the skin of the hand $0.5 \mathrm{~mm}$ for $400-$ $500 \mathrm{~ms}$. The probe was lifted off the skin for $2.0 \mathrm{~s}$ before it was lowered for another indentation. This sequence was repeated 100-150 times at each tested site. Only one stimulus probe was used in the conditions reported here, such that only one location on a single digit or palm pad was stimulated during a given recording. The purpose of stimulation in this study was to collect activity from as many neurons as possible from each monkey and calculate "response fields" for individual neurons under multiple, controlled stimulation conditions (see Data analysis). Stimulating different locations on the hand allowed us to drive firing above spontaneous rates for neurons with RFs across the hand, generating response fields for individual neurons and heat maps of activation across the array. Detailed responses of neurons in deafferented monkeys under these stimulation parameters and parameters using two stimulus probes will be addressed as part of a separate report.

$R F$ mapping. For each electrode, we determined neuronal RFs by light touch with fine probes, tapping, and joint movement. RF location, size, and stimulus preference at the site where the strongest evoked response occurred were documented. Neuronal responsiveness was classified into 
A

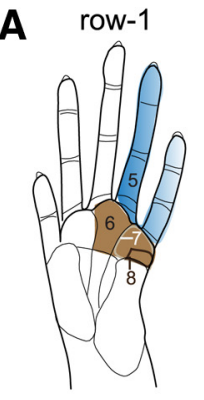

B

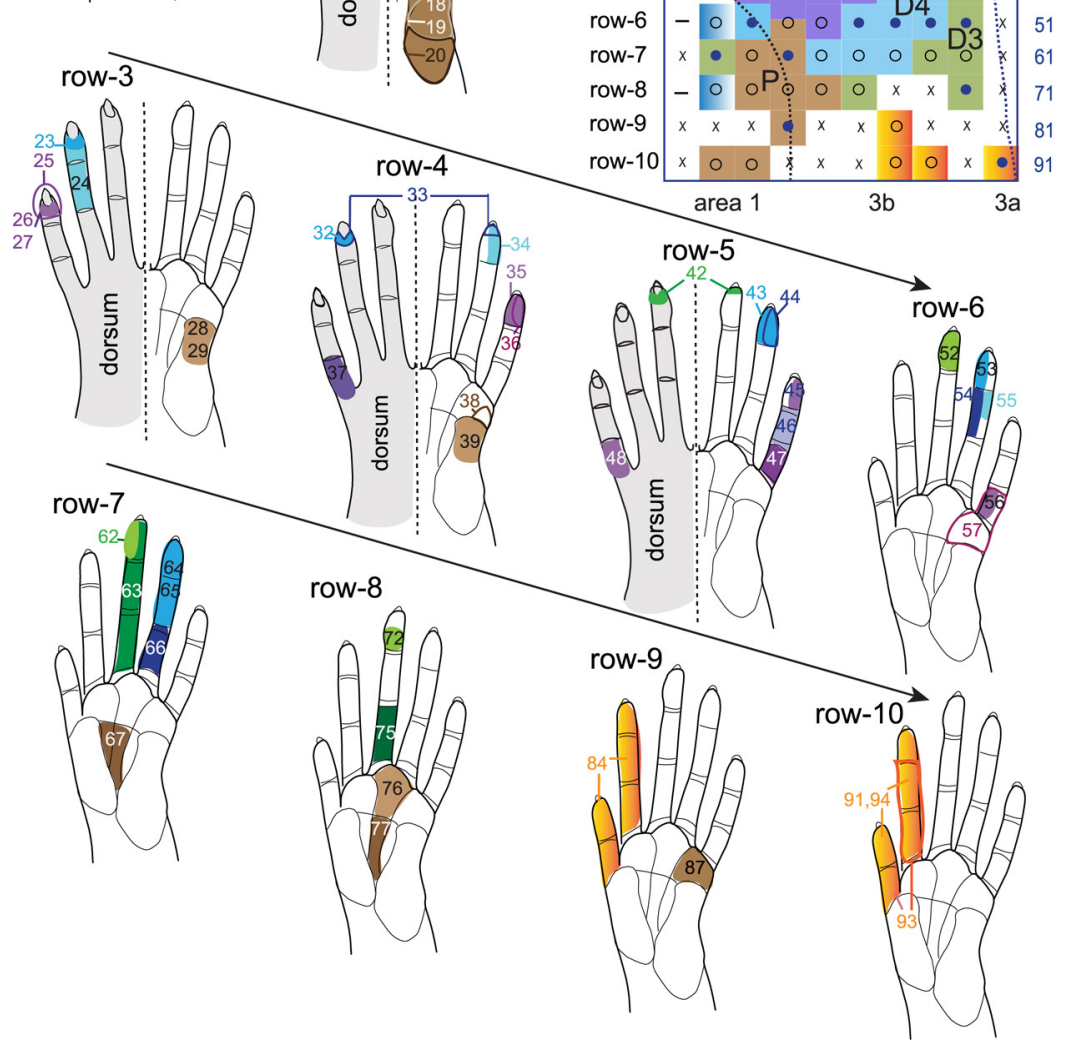

Figure 6. Organization of the hand representation of deafferented area $3 \mathrm{~b}$ in Monkey OM-M 5 weeks after lesion. $A$, Schematic drawings of hands represent neuronal RFs progressively shifted from distal to proximal portions of the hand, from the rostral to caudal direction of the array (rows 1-10) in the right hemisphere area 3b. B, Top, Schematic drawing of OM brain to show the location of the electrode array (square). Bottom, Somatotopic map was reconstructed based on the locations of RFs for neurons at each electrode. The lateral portion of the cortex representing digits 1 and 2 was mostly unresponsive. However, even with $<1 \%$ of afferents spared in the cuneate nucleus, digit representations $3-5$ responded well, and somatotopy was similar to that of normal monkeys. Nevertheless, unusually large RFs and over-represented dorsal hairy skin suggest abnormalities in area 3b. Solid dots indicate responses ranging from good to excellent at a given electrode; open circles represent weak responses; $x$ indicates microelectrode penetrations with no responses; "-" indicates disabled electrodes (in which recordings were turned off because of high levels of noise). Dashed lines indicate the rostral and caudal borders of area $3 \mathrm{~b}$ defined by topography and myelin architecture. 3a, Area 3a; 3b, area 3b; D3, D4, and D5, digits 3, 4, and 5, respectively; GR, good response; LS, lateral sulcus; M, medial; NR, no response; $P$, palm; $R$, rostral; STS, superior temporal sulcus; WR, weak response. $A$, Numbers indicate channel numbers.

across multiple recordings to standardize sorting assignments. We used Plexon Offline Sorter (Plexon) to verify the quality of unit isolation such that single units had refractory periods $\geq 1.2 \mathrm{~ms} ; p$ values $\leq 0.05$ for multivariate ANOVA related to cluster separation; and distinct waveform shapes and amplitudes when compared with other activity on the same electrode (Nicolelis et al., 2003). Single units and multiunits were categorized separately for comparisons of single-unit firing rates. But single units and multiunits were grouped together for factor analysis and illustrations.

Response field. We defined an excitatory "response field" as the region of skin where tactile stimulation increased peak firing rates within a $50 \mathrm{~ms}$ poststimulus time window for a given neuron (Reed et al., 2010a, b, 2011). Peak firing rate of each recorded neuron was first corrected by subtracting the average spontaneous firing rate of that neuron ( $500 \mathrm{~ms}$ before stimulus onset in PSTHs of at least 100 trials). Corrected peak firing rates of all recorded neurons were averaged to determine a population average firing rate and SD from multiple recordings. A single location on the hand was classified as inside a neuron's excitatory response field (IN) if the corrected peak firing rate of the individual neuron was at least 3 times greater than the SD of the population average firing rate. Otherwise, the skin location was considered outside of the response field (OUT). The criteria for IN the response field are therefore somewhat strict because firing rates of individual neurons must exceed the population SD. With this method, we use our simultaneous recordings such that firing during digit stimulation classified as IN the response field is more likely to be stimulusrelated rather than an increase in firing resulting from cofluctuations of activity across the electrode array. Calculations were performed using MATLAB (MathWorks) and exported to Excel (Microsoft). For visualization purposes, color maps of peak firing rates were generated for $50 \mathrm{~ms}$ windows after stimulus onset using MATLAB "colormap" commands ("hot"). In addition, we applied a 2D averaging filter ("average") from the Image Processing Toolbox with the default options (size $3 \times 3$ ) and assuming that values outside the bounds of the array are equal to the nearest border value ("replicate").

Digit territory ratio. We calculated response field ratios for neurons to stimulation on each digit, with the ratio $=\mathrm{IN} /(\mathrm{IN}+\mathrm{OUT})$. One

three categories: (1) cutaneous neurons responded to light contact on the skin or hair movement; (2) high threshold neurons required taps to the skin to evoke responses; and (3) noncutaneous, or "deep," neurons responded only to the manipulation of joints and muscles. Minimum RF was determined by the area of skin where light touches with a fine probe could activate neurons (Merzenich et al., 1978). RFs were recorded on a template of the hand or other relevant body part.

Data analysis

Spike sorting. The thresholded signals were sorted offline with an automatic spike classification program based on the $t$-distribution Expectation Maximization algorithm (Shoham et al., 2003). Details of the spike sorting procedures have been described previously (Reed et al., 2008; Reed et al., 2010b). Waveforms of action potentials were compared neuron was selected per electrode in area $3 \mathrm{~b}$ for ratio calculations. Because of variations in the array placement between monkeys, we recorded different numbers of neurons representing each digit across cases. We calculated a radial-to-ulnar digit territory ratio (R-U digit ratio) within each monkey defined as follows:

$\mathrm{R}-\mathrm{U}$ Digit Ratio $=\left[\mathrm{IN}_{\mathrm{R}} /\left(\mathrm{IN}_{\mathrm{R}}+\mathrm{OUT}_{\mathrm{R}}\right)\right] /$

$\left[\mathrm{IN}_{\mathrm{U}} /\left(\mathrm{IN}_{\mathrm{U}}+\mathrm{OUT}_{\mathrm{U}}\right)\right]$

This ratio complements estimates of digit territory from traditional mapping methods (e.g., Merzenich et al., 1978) and provides standardization to allow comparisons across cases. Ratio values $>1$ indicate that territory of a radial digit is larger than expected. 
Discontinuous response field ratio. We tallied the number of electrodes that recorded neuron activity in response to 0,1 , or 2 or more locations, summarized after individual digit or palm pads were stimulated with the mechanical probe. From this tally, we identified neuron activity evoked by discontinuous hand locations. For example, activity from one electrode may be responsive when a single probe stimulates D2 for 100 trials and when a probe stimulates D4, but unresponsive to D3 stimulation. We counted these discontinuous occurrences strictly. (For example, if we did not stimulate digit 3 during a recording session, then we did not count neuron responses from D2 and D4 stimulations as a "discontinuous response field.") The proportion of response fields that showed discontinuity (discontinuous RF) was calculated using the following ratio: number of discontinuous response fields divided by the total number of response fields that included more than one stimulation location. The utility of this measure is that it includes the firing rates of individual neurons as a parameter for estimating abnormality within the hand representation.

Statistics. Data were prepared in Excel and imported into IBM-SPSS 20.0 (IBM) or MATLAB for analysis. Unless otherwise stated, statistical significance levels were $\alpha=0.05$. Nonparametric statistical comparisons (Wilcoxon-Mann-Whitney two-sample rank-sum tests) between measures from normal and deprived monkeys were performed. Pearson's correlation coefficient and nonparametric correlation coefficients (Spearman's $\rho$ ) were calculated to determine whether significant relationships existed between behavioral impairment, axon sparing from the lesion, and neurophysiological measures of cortical reactivation and reorganization.

\section{Tissue processing and histology}

At the end of the terminal multielectrode recording session, each monkey was given a lethal dose of anesthetic (sodium pentobarbital, $120 \mathrm{mg} / \mathrm{kg}$, i.v.). When areflexive, monkeys were perfused transcardially with phosphate buffered $0.9 \%$ saline (PBS, $\mathrm{pH} 7.4$ ) followed by $2-4 \%$ PFA in phosphate buffer (PB), followed by $2-4 \%$ PFA with $10 \%$ sucrose in PB. The brain and spinal cord were separately removed. Cervical levels were defined before removal of the spinal cord from the vertebrae. The dura was cut along the midline from the junction of lower brainstem rostrally toward C8 caudally. The rootlets of each cervical segment were exposed to identify C1-C8. Pins were usually placed away from the lesion site at either the gap between two segments or in the centers of identified cervical segments.

This allowed us to determine the position of labeled afferents and the level of the lesion.

Cortex was separated from subcortical structures, manually flattened, and kept flat between glass slides (for progressive steps in flattening SM cortex, see Gharbawie et al., 2011). The cortex, brainstem, and spinal cord were stored overnight in $30 \%$ sucrose in PB for cryoprotection. The cortex was cut parallel to the surface into $40 \mu \mathrm{m}$ sections on a freezing microtome, and the sections were stained for myelin (Gallyas, 1979) to
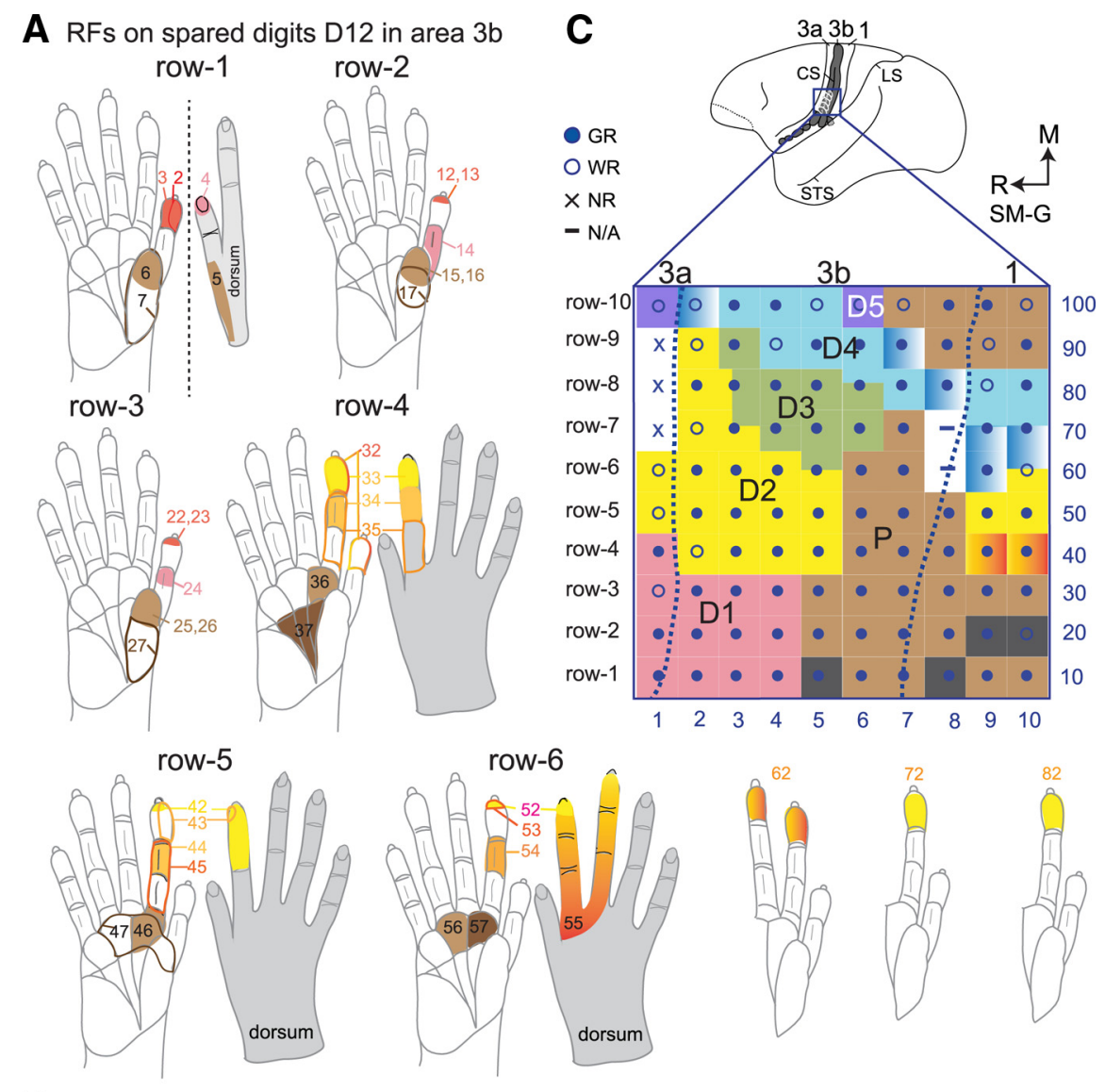

B RFs on partially deafferented digits D345 in area 3b
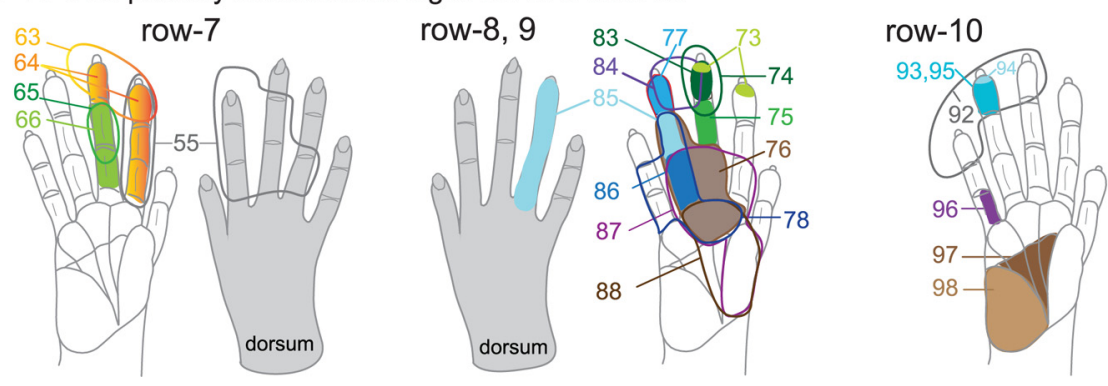

Figure 7. Organization of the hand representation of deafferented area $3 \mathrm{~b}$ in Monkey SM- $G 6$ weeks after lesion. $A$, Schematic drawings of hands represent neuronal RFs in largely spared digits 1 and 2 representations of area $3 \mathrm{~b}$. Distal to proximal representations of individual digits mapped onto the rostrocaudal orientation of the array (rows 1-10) in the left hemisphere area 3b. RF sizes within D1 and D2 representations were usually small (i.e., first 5 rows). $B$, Schematic drawings of hands represent neuronal RFs in deafferented digit representations $3-5$ of area $3 \mathrm{~b}$. RFs in this region were much larger and included multiple phalanges, multiple digits, or both glabrous and dorsal skin (e.g., electrodes 55, 63, 64, 66, 84, 85, 87, and 92, etc.). C, Top, Schematic drawing of a SM brain illustrates the location of electrode array (square). Bottom, Somatotopic map was reconstructed based on the locations of RFs for neurons at each electrode. The map demonstrates that digits $1-5$ are organized in the expected lateromedial progression, but the representations of D1 and D2 appear to be much larger than those of D3-D5. Solid dots indicate responses ranging from good to excellent at a given electrode; open circles represent weak responses; xindicates microelectrode penetrations with no responses; "-" indicates disabled electrodes. Dashed lines indicate the rostral and caudal borders of area $3 \mathrm{~b}$ defined by topography and myelin architecture. 1,3a, and 3b, Areas 1,3a, and 3b; CS, central sulcus; D1-D5, digits 1-5, respectively; GR, good response; LS, lateral sulcus; M, medial; NR, no response; P, palm; R, rostral; STS, superior temporal sulcus; WR, weak response. $\boldsymbol{A}$, Numbers indicate electrode numbers. 
A
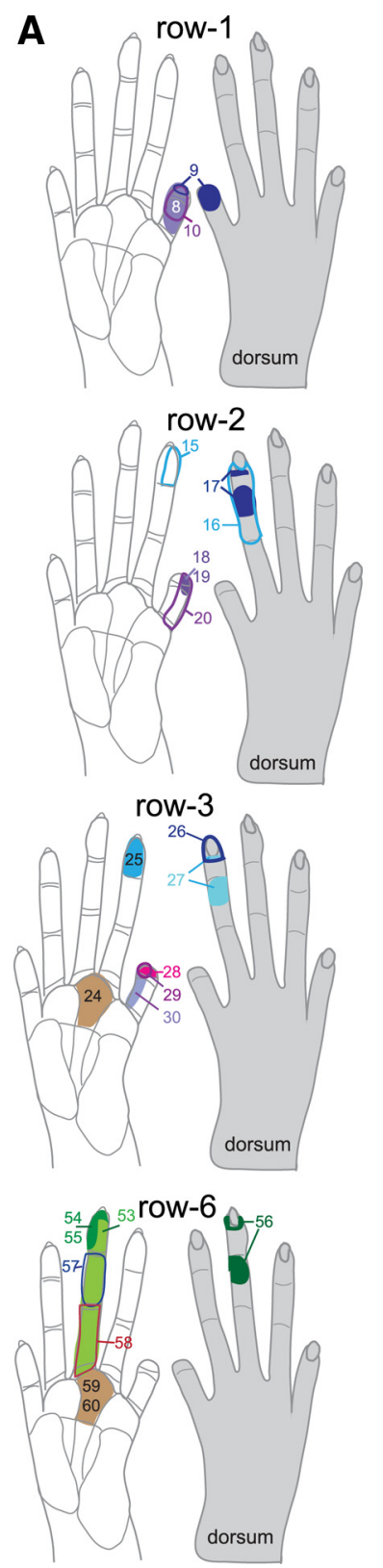

B

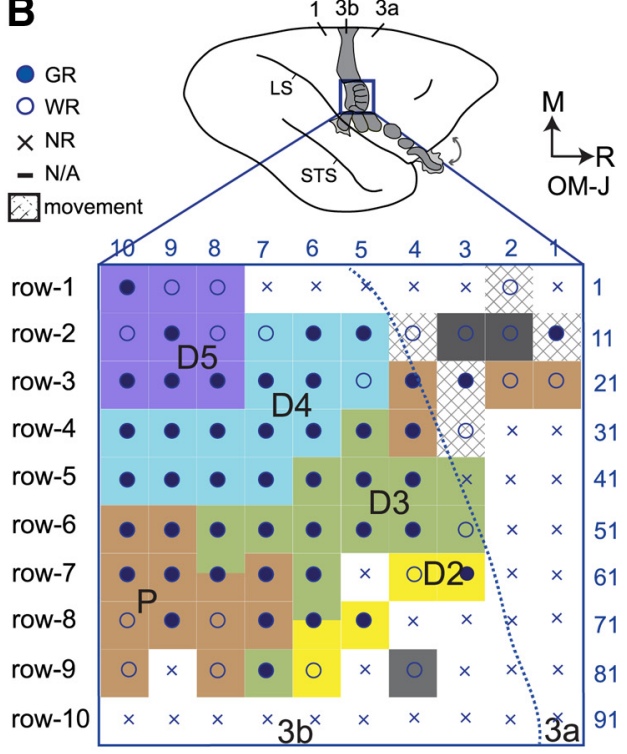

row-4
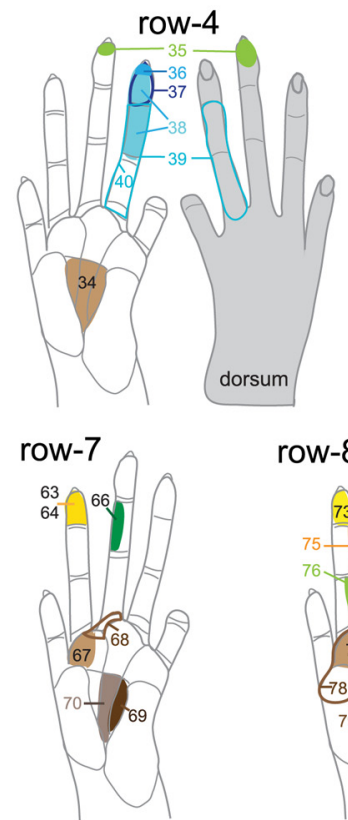

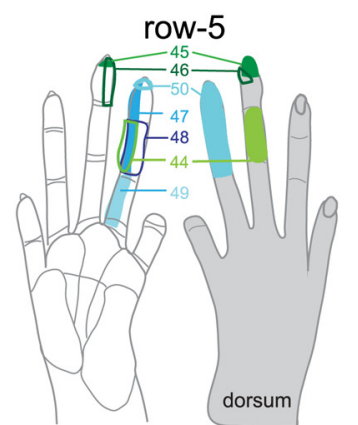

row-9 row-8

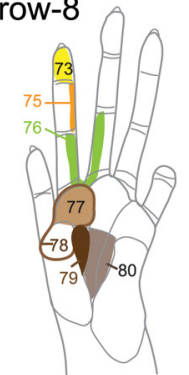

Figure 8. Organization of digit representations of partially deafferented somatosensory area $3 \mathrm{~b}$ in Monkey $0 \mathrm{M}-\mathrm{J} 10$ weeks after unilateral dorsal column lesion. $\boldsymbol{A}$, Schematic drawings of hands depict neuronal RFs progressively shifted from distal to proximal portions of the hand when electrodes were mapped across the rostrocaudal direction (rows 1-10) in area $3 \mathrm{~b}$ (right hemisphere). $B$, Top, Schematic drawing of an OM brain to illustrate the location of electrode array (square). Bottom, Somatotopic map was reconstructed based on locations of RFs for neurons at each electrode. The lateral portion of the cortex representing digit 1 was unresponsive, but digits $2-5$ were represented with somatotopy similar to normal monkeys. However, discontinuous RFs were found in multiple locations (e.g., electrodes 17, 27, and 56). Conventions follow Figures 6 and 7.

To reconstruct dorsal column lesions, images of the spinal cord sections were acquired using a Nikon E800 microscope (Nikon) and a Nikon DXM1200 camera (Nikon). Spinal cord sections were aligned to a pinhole along the midline. The maximal extent of the lesion, the white matter, and the gray matter were measured, and a coronal view of the lesion site was reconstructed from the series of sections with Adobe Illustrator software (Qi et al., 2011).

Lesion size measurement. Our lesion model was restricted to the dorsal column of the spinal cord; therefore, a measurement of total lesion volume was not applicable in the present study. We incorporated two methods here: (1) reconstruction of the damaged zones from spinal cord tissue (cut in a horizontal plane) to estimate cross-sectional area of the dorsal column for which axons were cut; and (2) estimation of the effective

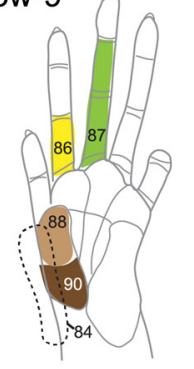

lesion by comparing distributions of tracerlabeled terminals (in the dorsal horn and cuneate nucleus) from axons that were spared from the lesion. From reconstructions of the lesion in each case projected into a coronal plane (Fig. 1), we used National Institutes of Health ImageJ software (http://rsbweb.nih.gov/ij/) to measure the cross-sectional area of the dorsal column for which axons were cut (lesion) compared with the cross-sectional area of the dorsal column at that cervical level (total DC area).

Quantification of CTB label. In addition to the reconstruction method to estimate the lesion size, to better quantify the effectiveness of the lesion, we used National Institutes of Health ImageJ software to measure the areas that contained CTB-labeled terminals throughout the cuneate nucleus. Detailed procedures have been described previously (Qi et al., 2011). All measurements from ImageJ were imported into Excel, and the ratio between the lesion side and intact side was calculated (for details, see Qi et al., 2011). In Monkey SM-G, two different tracers were subcutaneously injected into the left hand (CTB) and right hand (BHRP). The purpose of this deviation was to determine whether peripheral afferents projected to both sides of the cuneate nucleus. Because two different tracers were injected into left and right hands, we used the following formula for Monkey SM-G:

Ratio (Lesion vs Intact) $=[$ (Labeled

area/total area on Lesion side)/(Labeled

area/total area on Intact side) $] \times 100$.

Statistical comparisons between measures of the lesion and intact sides of sections were performed in MATLAB (Wilcoxon matched-pairs signed-ranks test, $\alpha=0.05$ ).

\section{Results}

The primary goal of this study was to investigate how dorsal column lesions at the midcervical spinal cord (C4-C6) affect neuronal response properties in contralateral primary somatosensory cortex, area 3b. We also examined extent of DC lesion, behavioral recovery, and cortical reactivation to determine the relationship between all three factors. The results are presented in 5 sections: (1) extent of lesion; (2) behavioral performance; (3) somatotopy and neuronal RF properties after behavioral recovery; (4) quantitative characterization of response fields after behavioral recovery; and (5) relationships between axon sparing, behavioral recovery, and responsiveness of cortex within monkeys, including comparisons of response characteristics between normal and lesion monkeys. As the extents and levels of the lesions varied across monkeys, the results are largely presented on a case-by-case basis.

\section{Level and extent of DC lesion}

DC lesions were reconstructed for all 5 cases (Fig. 1). We found a somatotopic organization for afferents labeled in the spinal cord from tracer injections into the digits (Florence et al., 1989, 1991). 
For example, injections into D1 labeled afferents near $\mathrm{C} 5$, whereas injections into D3 and D5 labeled afferents near C6 and $\mathrm{C} 7$, respectively. In most cases (4 of 5), lesions were restricted to the dorsal columns and some of the central gray of the spinal cord. The corticospinal tract, one of the major motor pathways involved in dexterity, was spared in all cases, except in Monkey SM-G.

\section{Monkey SM-G}

The dorsal column lesion was extensive in this case but at a slightly lower cervical level than all other monkeys. The lesion encroached into the dorsal horn, intermediate zone, portions of the ventral horn, and corticospinal tract (Fig. 1). To determine whether peripheral afferents projected to the nuclei on the same side only (ipsilateral) or on both sides (bilateral), we subcutaneously injected two different tracers into the left hand (CTB) and right hand (BHRP) in this case (Fig. $2 A, B$ ). We did not detect bilateral projections in either spinal cord or brainstem. Therefore, in all other cases, we injected CTB in both hands to facilitate comparison of labeling in both sides of the cuneate nucleus. In Monkey SM-G, BHRP tracer was injected into all 5 digits of the deafferented hand. Fiber terminals were labeled throughout the dorsal horn in C5-C7 and the lesion was at C6 (Fig. 2B). Thus, peripheral afferents from digits 1 and 2 were largely spared, and afferents from D3-D5 were mostly cut. In the cuneate nucleus, patches of BHRP-labeled axons and terminals can be identified throughout multiple sections, but they were sparsely distributed. The locations of those sparse patches likely reflected preserved inputs from digits 1 and 2. CTB was injected in the distal phalanges of digits $1,2,3$, and 5 of the intact hand. Dense foci of axon terminal fields were apparent in the spinal cord and cuneate nucleus.

On the lesioned side, we measured BHRP-labeled areas and the total area of cuneate nucleus from the same sections to determine the proportion of area labeled in cuneate. This procedure was followed for the intact side (Fig. 2C). Finally, the ratio of both proportions showed that $\sim 20.7 \%$ of axons were present in the ipsilateral-tolesion cuneate nucleus compared with the opposite cuneate nucleus (Fig. 2D). This represents a significant reduction on the lesion side $\left(p=1.17 \times 10^{-6}\right.$, Wilcoxon matched-pairs signedranks test, $Z=4.86, n=31$ sections).

\section{Monkey OM-M}

CTB was injected into matched locations in the distal and middle phalanges of digits 3 and 4 on both hands. Lesion reconstruction showed that it was at the C4 level on the left side of the spinal cord, which suggests that peripheral inputs from all digits of left hand were interrupted (Fig. 3A,B). Densely labeled axon terminals

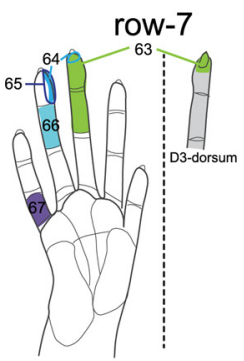

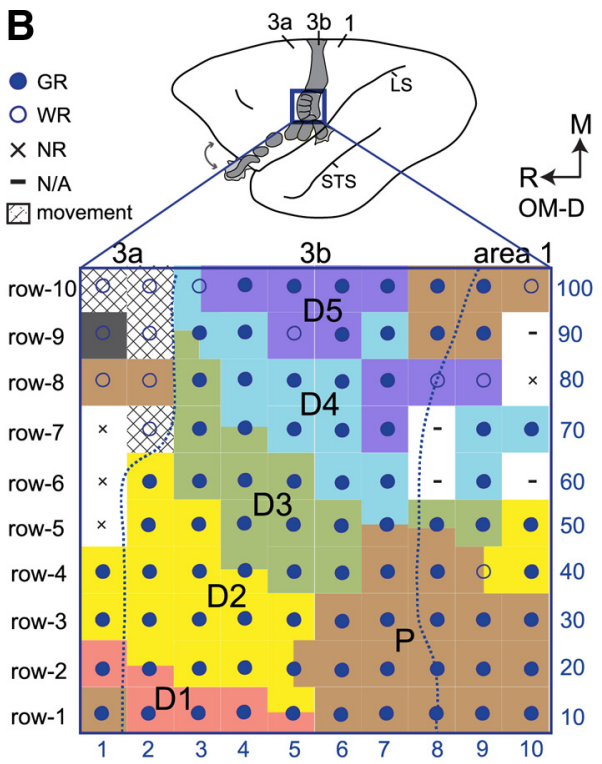
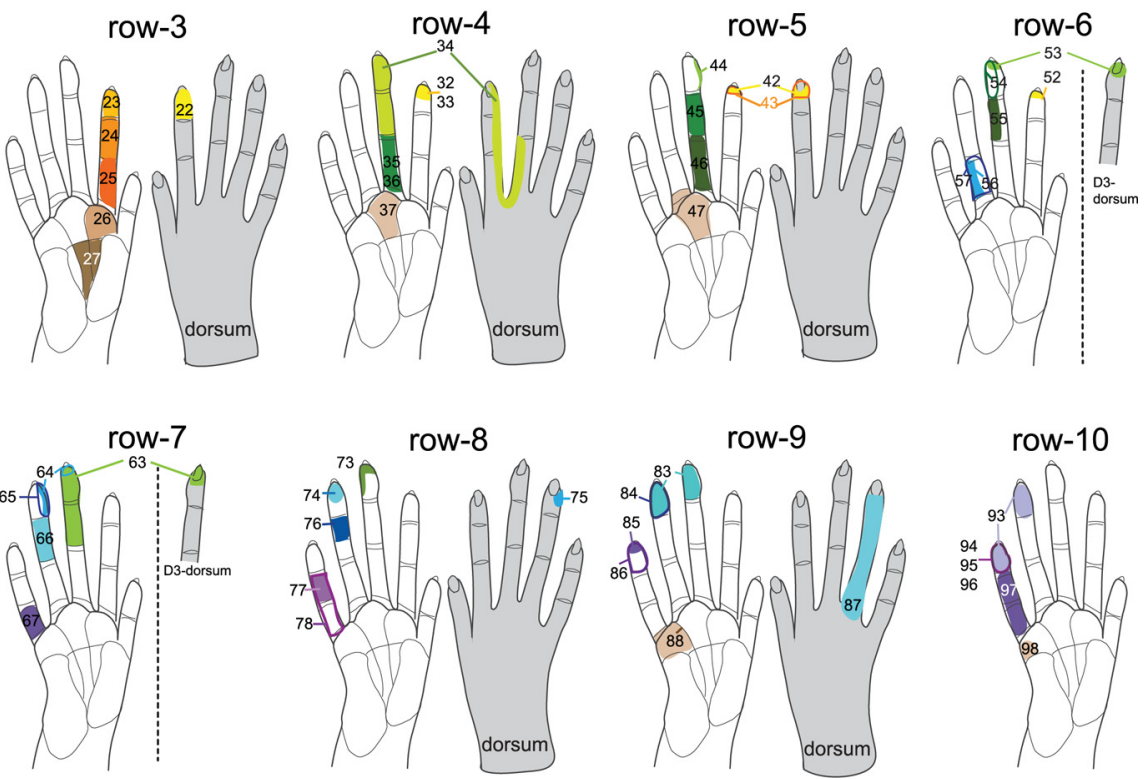

Figure 9. Organization of digit representations of partially deafferented somatosensory area 3b in Monkey 0M-D 7 weeks after unilateral dorsal column lesion. $A$, Neuronal RFs progressively shifted from distal to proximal portions of the hand when electrodes (rows 1-10) were mapped from the rostrocaudal direction in area 3b (right hemisphere). $\boldsymbol{B}$, Top, Schematic drawing of an 0M brain to show the location of electrode array (square). Bottom, Somatotopic map was reconstructed based on locations of RFs for neurons at each electrode. With $\sim 50 \%$ of spared fibers reaching the cuneate nucleus, the somatotopy, responsiveness, and the RF sizes resembled those of normal monkeys. Conventions follow Figures 6 and 7.

were identified across many sections throughout the intact cuneate nucleus. In contrast, labeled terminals were sparse and present in only a few sections of the deafferented cuneate (Fig. 3C). The approximate area of labeled terminals on the lesion side was $\sim 0.56 \%$ of the intact counterpart (Fig. $4 A$ ), and this difference was significant $\left(p=1.11 \times 10^{-8}\right.$, Wilcoxon matched-pairs signed-ranks test, $Z=5.71, n=43$ sections). These results suggest that the lesion interrupted $>99 \%$ of the inputs from digits 3 and 4 . Along with the evaluation of the lesion from the spinal cord reconstruction, the results suggest that inputs from all digits of the hand were severely deafferented (Fig. 3).

Restricted DC lesions were found in Monkeys OM-J, OM-D, and SM-Ro. Monkey OM-D appeared to have some sparing of axons in the cuneate fasciculus (Fig. 1). The reconstruction of 
A
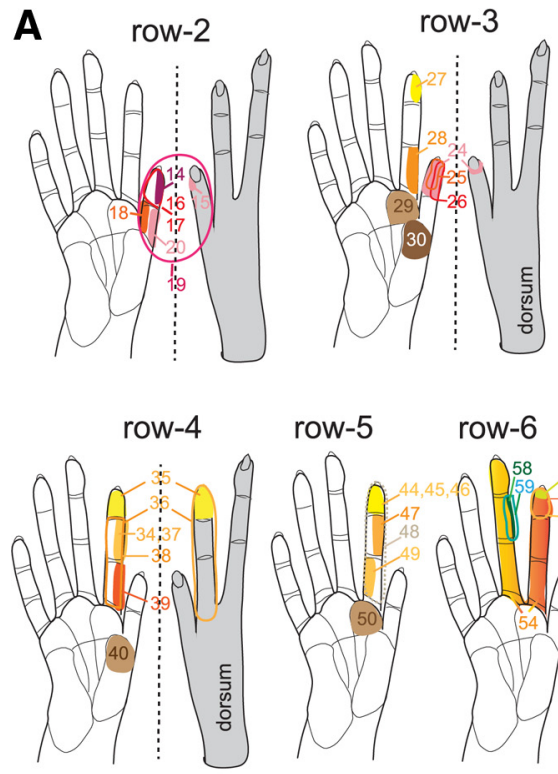

row-5

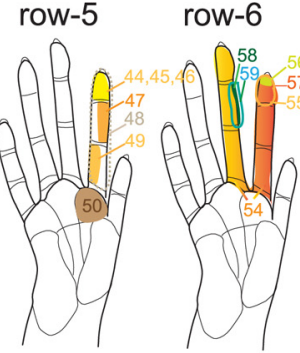

row-7
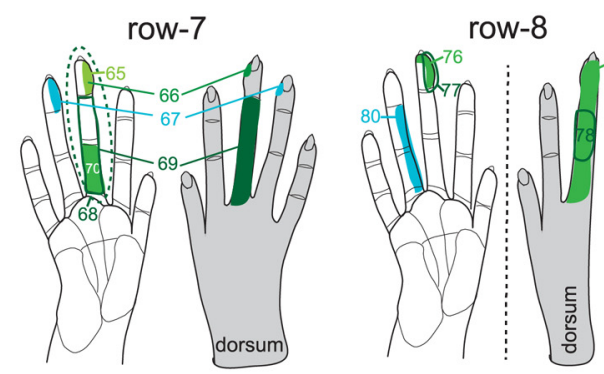

8
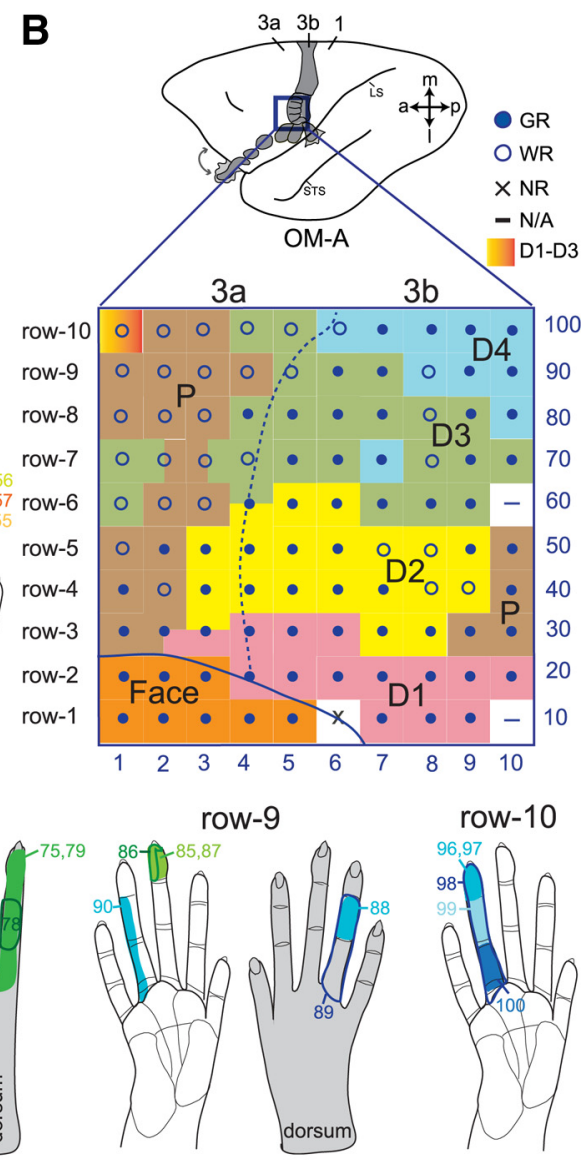

C
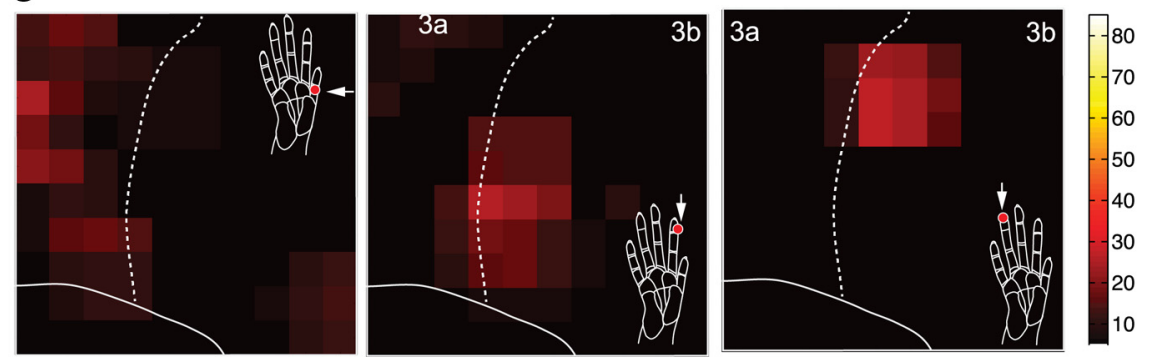

Figure 10. Organization of the hand representation of area $3 \mathrm{~b}$ in a normal Monkey OM-A. A, Schematic drawings of hands represent neuronal RFs progressively shifted from distal to proximal portions of the hand, from the rostral to caudal direction of the array (rows 1-10) in the left hemisphere area $3 \mathrm{~b}$ hand region. $\boldsymbol{B}$, Top, Schematic drawing of $0 \mathrm{M}$ brain representing the location of the electrode array (square). Bottom, Somatotopic map was reconstructed based on the locations of RFs for neurons at each electrode. Conventions follow Figures 6 and 7 . The somatotopy was similar to that of previous reports from normal monkeys. $C$, Heat maps of the peak firing rate responses (50 ms response windows) across the array were generated in MATLAB with an averaging filter (size $=3$ ) and hot colors representing higher peak firing rates (color scale $5-85$ spikes $/ \mathrm{s}$ ). Dashed lines indicate rostral and caudal borders of area $3 \mathrm{~b}$. Peak firing rate responses were represented in expected somatotopic order. Visualization is an average map in which the firing of neurons from points surrounding each square contribute to the color code (filter size $=3$ ) rather than a point-to-point match for all neurons recorded from each electrode. Inset (into each heat map), Schematics of the OM hand represent tactile stimulation sites (red dot) on individual digits (arrow).

lesion extent in Monkey OM-J indicated that the lesion was restricted to a portion of cuneate fasciculus in the dorsal column (Fig. 1). The lesion in Monkey SM-Ro encroached into the dorsal horn, intermediate zone, and portions of the ventral horn, but a fair amount of fibers in the medial portion of the DC was spared (Fig. 1).

In these 3 monkeys, CTB tracer was injected into digits 1, 3, and 5 at matched locations of both hands. CTB labeling indicated that the lesion interrupted peripheral inputs from all digits of the left hand at the C4 level in all 3 monkeys. In the cuneate nuclei,

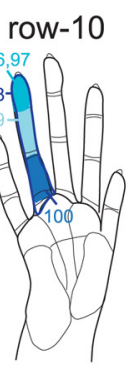

labeled fields occupied a smaller territory on the lesion side compared with the intact side. Axons spared on the lesion side amounted to $\sim 46.5 \%$ in Monkey OM-J (Fig. 4B), 49.6\% in Monkey OM-D (Fig. $4 C$ ), and $59.5 \%$ in Monkey SM-Ro (Fig. $4 D$ ) of axons on the intact side. Areal differences between the two sides were significant in all 3 cases (OM-J: $p=8.32 \times$ $10^{-5}$, Wilcoxon matched-pairs signedranks test, $Z=3.94, n=51$ sections; OM-D: $p=3.95 \times 10^{-4}, Z=3.54, n=26$ sections; SM-Ro: $p=2.05 \times 10^{-5}, Z=$ $4.26, n=42$ sections).

\section{Behavioral impairment after spinal cord lesion}

After DC lesion, monkeys often tried to perform the task with the unaffected hand. When that happened, we discouraged those attempts by blocking the hand or covering the pellets manually. Then, monkeys learned to use the impaired hand to retrieve pellets. Monkeys required different amounts of recovery time before using the impaired hand. The postlesion day when we observed use of the impaired hand reflected impairment magnitude. From the reach-to-grasp task with the impaired hand, we compared mean success scores and number of flexes before and after lesion to assess performance during individual testing weeks after lesion. Cases are presented in the order of estimated axon sparing.

\section{Monkey OM-M}

This monkey had a nearly complete C4 lesion and did not use the impaired hand to retrieve food items until day 21 after lesion. Although performance was not quantitatively assessed for this monkey, we consistently observed the hand approach the mouth regardless of whether a food item was grasped. This behavior suggests that the monkey was "unaware" if indeed the food pellet was secured in its hand, which was not the case before lesion.

\section{Monkey SM-G}

This monkey was unable to perform the reach-to-grasp task with the impaired hand up to day 5 after an extensive DC lesion at C6. In the first week after lesion, mean success scores tended to decline, but Dunn's multiple comparisons tests did not reach statistical significance. In subsequent weeks, mean success scores returned to levels recorded before lesion (Fig. 5A). However, success scores for the most difficult well were not stable and further declined 3 weeks after lesion (Kruskal-Wallis $=27.380$, $p=0.0003, N=655$ observations, Dunn's $p<0.001$ ). In addition, the increase in the number of digit flexes suggested that pellets in wells 3 and 4 were more difficult to access than pellets in 
wells 1 and 2 (Fig. 5B), and these effects lasted up to 5 weeks after lesion (KruskalWallis $=37.055, p<0.0001, N=655$ observations, Dunn's $p<0.001)$.

\section{Monkey OM-J}

This monkey was able to perform the reach-to-grasp task with the impaired hand $1 \mathrm{~d}$ after a restricted $\mathrm{C} 4$ lesion. Performance levels were maintained up to day 56 and were not distinguishable from prelesion levels (Kruskal-Wallis $=2.323$, $p=0.5082, N=155$ observations tested).

\section{Monkey OM-D}

This monkey was unable to perform the task with its impaired hand up to $6 \mathrm{~d}$ after a restricted C4 lesion. Dorsal column lesion increased the number of flexes for wells 3 and 4 during week 2 testing compared with prelesion levels (well 3: Kruskal-Wallis $=36.896, p<0.0001$, $N=311$ observations tested; well 4: Kruskal-Wallis $=11.658, p=0.0398$, $N=268$ observations tested). Performance recovered to prelesion levels by week 5 (well 3: Kruskal-Wallis = 2.694, $p=0.7471$; well 4: Kruskal-Wallis = 2.846, $p=0.7237)$.

\section{Monkey SM-Ro}

We were unable to train this SM to accept sugar pellets; however, we filmed its reach-to-grasp behavior retrieving pieces of raisins in a similar variation of the standard task. This monkey was able to perform the task with its impaired hand within $2 \mathrm{~d}$ of a restricted $\mathrm{C} 4$ lesion and showed no obvious deficits.

We ranked the performance of the lesion monkeys to relate their dexterity to lesion extent and measures of cortical reactivation. The first category we considered was the number of days after lesion in which the monkey was unable to grasp with the impaired hand. Monkey OM-M was ranked as most impaired because it was unable to retrieve small food items from the testing board for 2 weeks after lesion. This impairment lasted much longer than any other monkey. We ranked the remaining cases using two additional categories: mean difference between postlesion and prelesion values in total digit flexes and mean difference in success score of the last testing day after lesion versus before lesion. In this way, initial impairment and recovery on the last testing day were included in this measure to relate better to the endpoint stage of the electrophysiological and anatomical analyses. The final rank was calculated from the average of the three categories. Thus, we ranked the lesioned monkeys from most to least impaired as follows: Monkeys OM-M, SM-G, OM-D, SM-Ro, OM-J (Table 1).

Estimates of lesion size based on reconstruction of the damaged tissue and based on the tracer labeling quantification are included in Table 1 to aid comparisons of the lesions with the behavioral deficit ranks. We found that the ranking of behavioral measures closely corresponded to the estimates of axon sparing
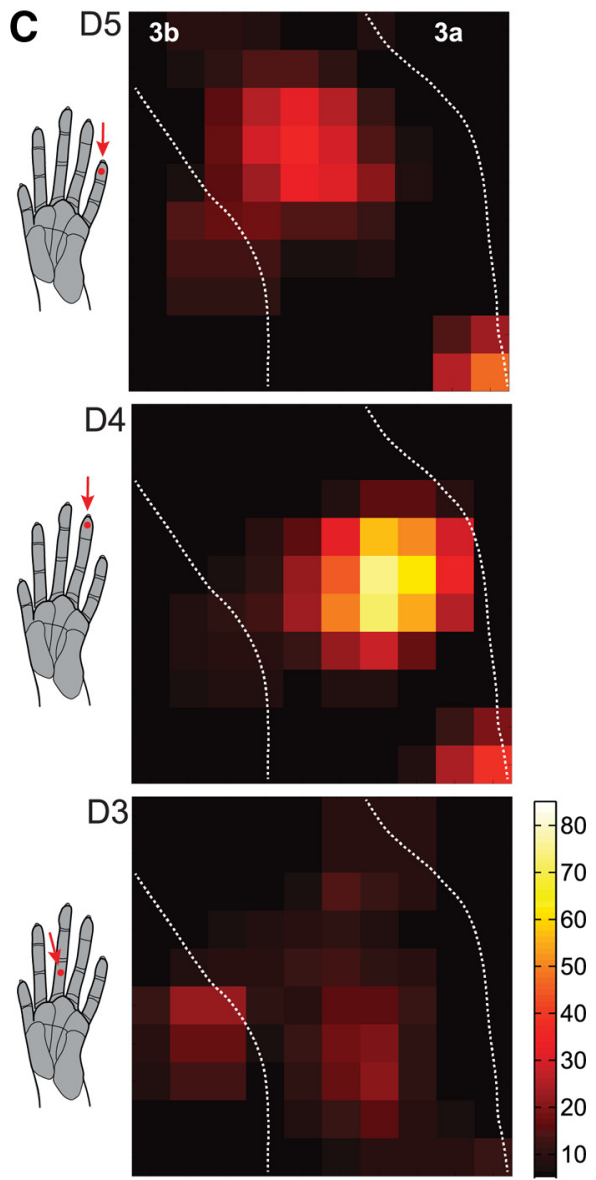

Figure 11. Responsiveness in partially deprived area 3b in Monkey 0M-M. A, A transverse view of spinal cord showing the extent of lesion (black shading) reconstructed from a series of horizontally cut spinal cord sections. $\boldsymbol{B}$, Photomicrograph from a myelin-stained section cut parallel to pia surface through somatosensory areas $3 \mathrm{~b}$ and 1 representing the array location (top) and firing rate activations in response to digit stimulation are relatively weak but are generally consistent with digit territories determined from RF mapping. Dashed lines indicate the rostral and caudal borders of area $3 \mathrm{~b}$ defined by topography and myelin architecture. Conventions follow Figures 6 and 7.

(Pearson's $r=0.8627 ; p=0.0598, n=5$ deprived monkeys), as shown in Figure $5 C$.

\section{Somatotopy and RF properties from neurons in partially deprived area $3 b$}

Five to 10 weeks after DC lesions, the deprived somatosensory areas $3 \mathrm{~b}$ and 1 were mapped. In each monkey, the responsiveness, neuronal RF sizes, and locations were characterized systematically from each electrode after array insertion. The somatotopic map was reconfirmed a day later if time allowed. For somatotopic reconstructions, minimum RFs were determined based on the best responses obtained for each electrode (for details of reconstruction, see Nelson et al., 1980; Sur et al., 1982; Kaas et al., 1983; Merzenich et al., 1983a,b). Areal boundaries were determined anatomically and electrophysiologically. Our criteria were based on studies showing that neurons in area $3 \mathrm{~b}$ have the smallest RFs in somatosensory cortex, low threshold responses, and a somatotopic organization that mirrors the topography of area 1 (Nelson et al., 1980; Sur et al., 1982; Kaas et al., 1983; Merzenich et al., 1983a,b). 
A

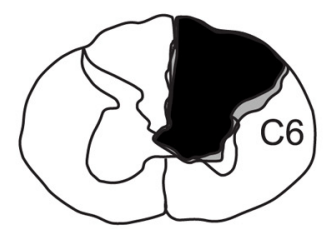

B
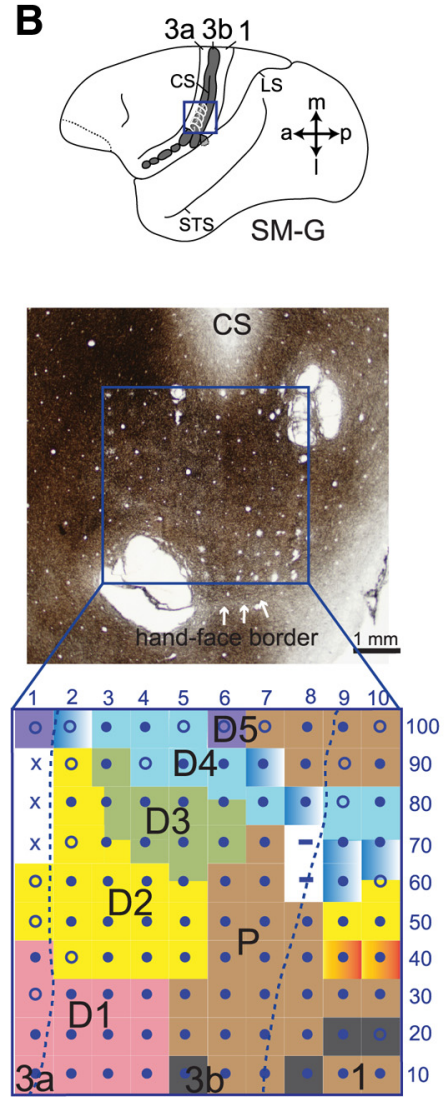

D1-D3 D3-D5

Hand, wrist, distal forearm
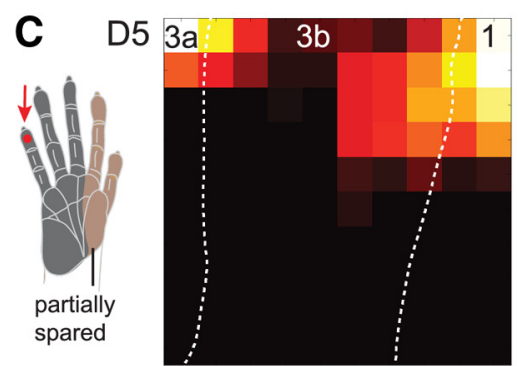

D4
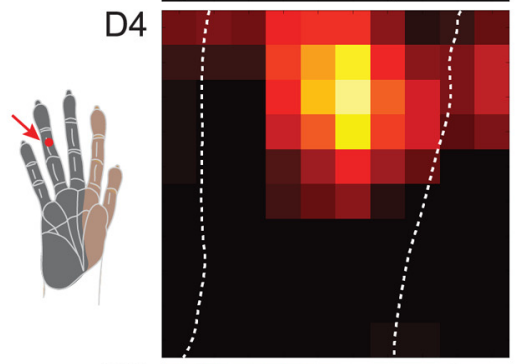

D3
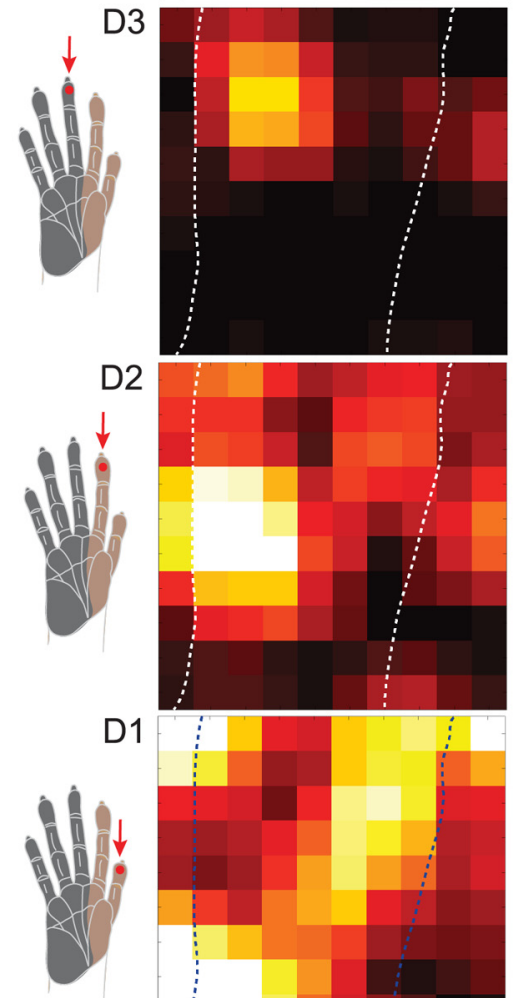

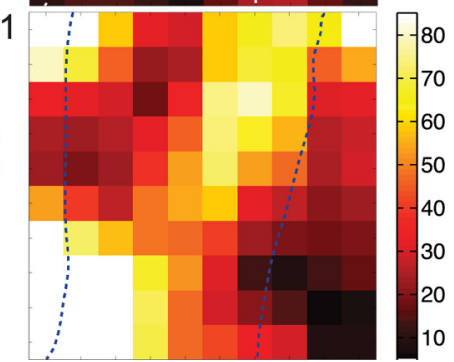

Figure 12. Responsiveness in partially deprived area $3 \mathrm{~b}$ in Monkey SM-G. $A$, A transverse view of spinal cord showing the extent of lesion (black shading) reconstructed from a series of horizontally cut spinal cord sections. $\boldsymbol{B}$, Top, Schematic drawing represents an overview of a SM brain. The array location is indicated by a box. Middle, Photomicrograph from a myelin-stained section cut parallel to pia surface through somatosensory areas $3 a, 3 b$, and 1 showing the array location. Bottom, Schematic drawing of a reconstructed somatotopic map. $C$, Heat maps of the peak firing rate responses across the array follow conventions of Figures 10 and 11 (color scale 5-85 spikes/s). Schematic drawings of SM hands represent the sites for tactile stimulation (red dots with arrows) on individual digits. Peak firing rate responses in mostly spared territories of $D 1$ and D2 were strong and extensive, and digits were represented in expected somatotopic order; however, electrodes in deprived representations (e.g., D4 and D5) recorded neurons responding strongly to stimulation on D1 and D2. $B$, " $x$ " indicates no response to light to moderate touch during minimal RF mapping but does not preclude the possibility of driven activity under the suprathreshold stimulation conditions using the computer-controlled mechanical probe or high levels of spontaneous activity. $C$, Average maps in which the firing of neurons from points surrounding each square contribute to the color code (filter size $=3$ ) rather than a point-to-point match for all neurons recorded from each electrode. Dashed lines indicate rostral and caudal borders of area $3 \mathrm{~b}$.

Monkey OM-M

The deprived area $3 \mathrm{~b}$ was mapped 5 weeks after an extensive lesion of the contralateral (left) dorsal columns at the C4 level. The electrode array was placed in the center of the hand representation in area $3 \mathrm{~b}$ (Fig. 6). Neurons in area $3 \mathrm{~b}$ responded to tactile stimulation on the digits (Fig. $6 A, B)$, even with only $0.56 \%$ of DC afferents remaining from digits 3 and 4 (Figs. 3 and $4 A$ ). Nearly all primary afferents in the dorsal columns from digits 1,2 , and 5 were also likely cut, but RFs were still mapped on these digits. We found that the lateral part of the hand representation in area $3 \mathrm{~b}$ did not respond to somatosensory stimuli, and neuronal responses in some electrodes were weak. Abnormally large RFs (e.g., ch. 5, 14, 84) and overrepresentations of dorsal skin were also apparent (e.g., electrodes in rows 2-5). Nevertheless, the medial part of the $3 \mathrm{~b}$ hand representation responded well to tactile stimulation, and digits D3-D5 were represented in a normal lateromedial somatotopic sequence (Fig. 6A).

\section{Monkey SM-G}

The deprived area $3 \mathrm{~b}$ was mapped 6 weeks after an incomplete lesion of the contralateral (right) dorsal columns. The electrode array covered the entire hand representation in area $3 \mathrm{~b}$. A number of electrodes were located in area $3 \mathrm{a}$ and area 1. Quantitative measurements indicated that $<20.7 \%$ of the primary afferents were spared (Fig. 2). Nevertheless, the location of the lesion at midcervical spinal cord level suggests that most of the inputs from digits 1 and 2 were spared. Weeks after this extensive but incomplete loss of afferents, the lateral parts of the hand representations in area $3 \mathrm{~b}$ responded well to tactile stimulation of the radial hand (Fig. 7). Moreover, the more deprived medial parts of the hand representation were also responsive to stimulation of the ulnar hand. Thus, all five digits were represented in normal lateromedial sequence from D1 to D5. The RFs of the largely spared digits 1 and 2 were small (Fig. 7A), resembling normal RF sizes in area $3 \mathrm{~b}$. In contrast, the RFs of neurons in the deprived representations of digits 3-5 were relatively large and usually included multiple digits or phalanges (Fig. $7 B$ ). For example, some of the RFs covered more than one digit (ch. 73, 76, 78, 84, 87, 92), some covered digit and palm pads (ch. 76,78 ), and some even covered both glabrous skin and dorsal skin (ch. 85). Overall, the responses from the electrode array indicate that the representation of digit 2 and, to a 
lesser extent, digit 1 were unusually large, suggesting that they had expanded into deprived digits 3-5 region of cortex (Fig. 7C).

\section{Monkey OM-J}

The deprived area $3 \mathrm{~b}$ was mapped 10 weeks after an incomplete lesion of the contralateral (left) dorsal columns. A larger proportion of afferents were spared (46.5\%) compared with the two monkeys discussed above (Fig. 4B). The hand representation in area $3 \mathrm{~b}$ responded well to tactile stimuli, except the D1 territory, which was largely unresponsive (Fig. $8 A, B)$. Digits $2-4$ were represented in the expected lateromedial sequence. RF sizes were abnormally large in several electrodes (e.g., electrodes 39, 53, and 87). Some RFs were in unusual locations, for example, neurons recorded on the same electrode responded to stimulation of discontinuous skin surfaces (Fig. 8A; electrodes 17, 27, and 56). This phenomenon is characterized in later sections.

\section{Monkey OM-D}

The deprived area $3 \mathrm{~b}$ was mapped 7 weeks after incomplete lesion of the contralateral (right) dorsal columns. The electrode array was placed in the center of the hand representation in area $3 \mathrm{~b}$ (Fig. 9B). The lesion extended more laterally into the dorsal horn at the C4 level, and higher proportions of afferents were spared than in the previous cases (49.6\%; Fig. 4C). The hand representation in area $3 \mathrm{~b}$ responded well to tactile stimuli. The representations of digits 1-5 followed the expected lateromedial sequence (Fig. 9A,B). RF sizes were comparable with normal monkeys.

\section{Monkey SM-Ro}

The deprived area $3 \mathrm{~b}$ was mapped 8 weeks after incomplete lesion of the contralateral (left) dorsal columns. The electrode array was placed mostly in the hand representation of area 1 to avoid damaging large blood vessels in area $3 \mathrm{~b}$. Nevertheless, 20 electrodes were located in the hand representation of area $3 \mathrm{~b}$ (data not shown). The lesion was at the C4 level and spared $59.6 \%$ of peripheral afferents (Fig. 4D). Partially deprived areas $3 \mathrm{~b}$ and 1 responded well to tactile stimuli. However, RFs for most electrodes were relatively large and showed disorganized somatotopy, including intermingling of representations of digits 2-4.

\section{Monkey OM-A}

For comparison with DC lesion monkeys, we describe RF mapping information for a normal case, Monkey OM-A (Fig. 10A). The electrode array was placed in the digit representation of area $3 \mathrm{~b}$ but included some of the representation of the face as well as area 3a (Fig. 10B). Missing from the array coverage were the
A

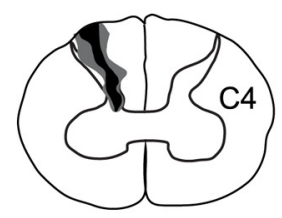

B
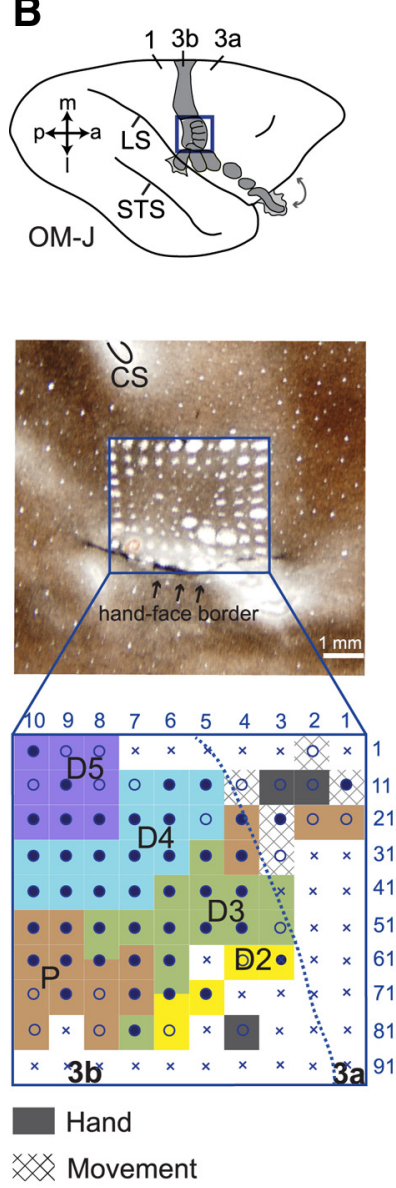
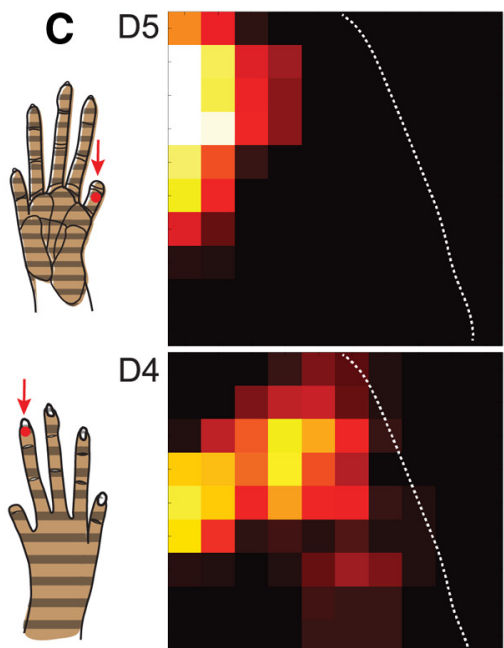

04
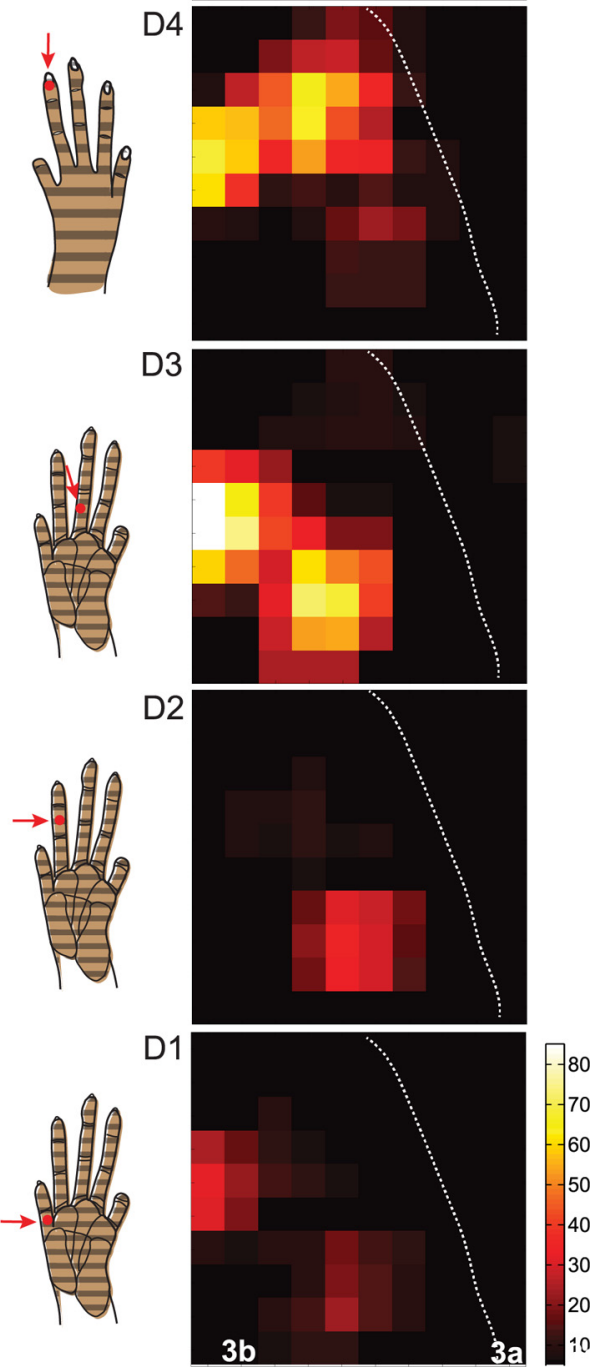

Figure 13. Responsiveness in partially deprived area 3b in Monkey 0M-J.A,A transverse view of spinal cord showing the extent of lesion (black shading) reconstructed from a series of horizontally cut spinal cord sections. $\boldsymbol{B}$, Top, Schematic drawing represents an overview of an $0 \mathrm{M}$ brain. The array location is indicated by a box. Middle, Photomicrograph from a myelin-stained section cut parallel to pia surface through somatosensory areas $3 \mathrm{~b}$ and 1 showing the array location. Bottom, Schematic drawing showing a reconstructed somatotopic map from array. $C$, Heat maps of the peak firing rate responses across the array follow conventions of Figures 10-12 (color scale 5-85 spikes/s). Schematic drawings of $0 \mathrm{M}$ hands indicate the sites for tactile stimulation (red dots with arrows) on individual digits. Stripes on hands indicate partial deafferentation. Peak firing rate responses were somewhat weak and restricted, but digit activations were represented in expected somatotopic order within territories determined from RF mapping. Dashed lines indicate rostral and caudal borders of area 3b. Conventions follow Figures 6 and 7.

center of the digit 5 representation and parts of the palm. RFs were contained within a single digit or palm pad, often confined to a single phalanx within a digit. Neurons typically responded well to light tactile stimulation, and the digits were represented in expected somatotopic order. These results are consistent with many previous studies of primary somatosensory cortex (e.g., Merzenich et al., 1978; Sur et al., 1982), and the somatotopic organization and response properties of neurons from this mon- 
A

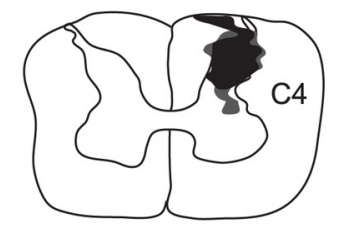

B
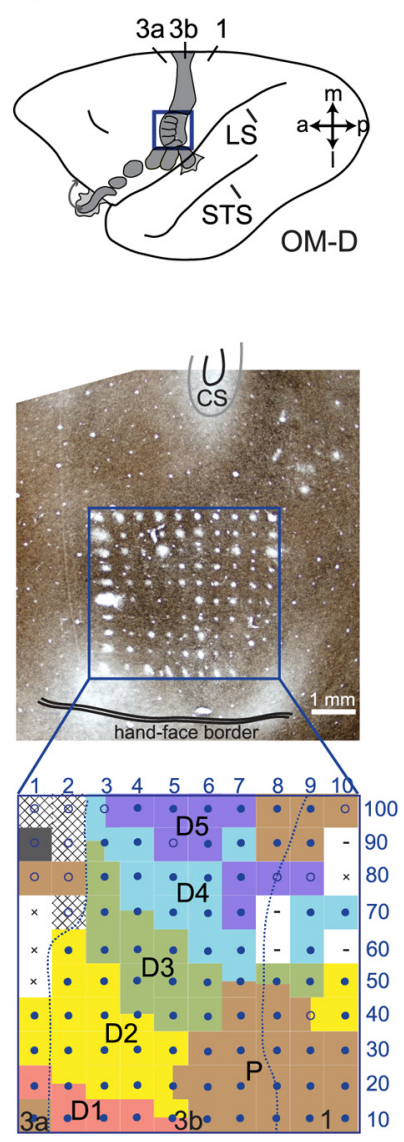

Hand $x \times x$ Movement
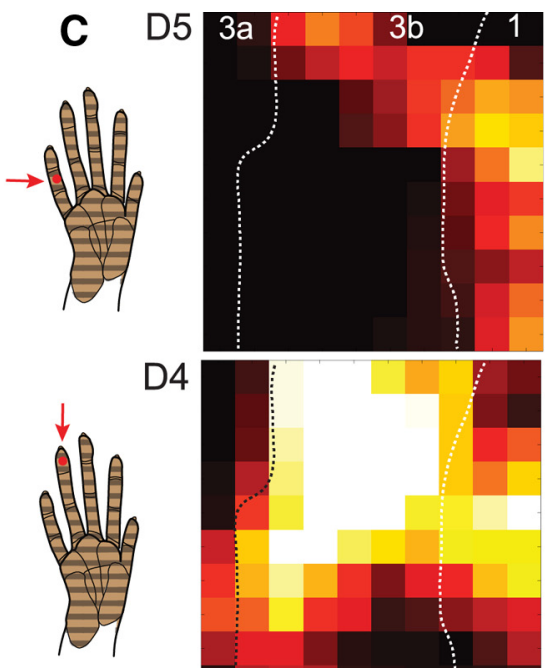

4
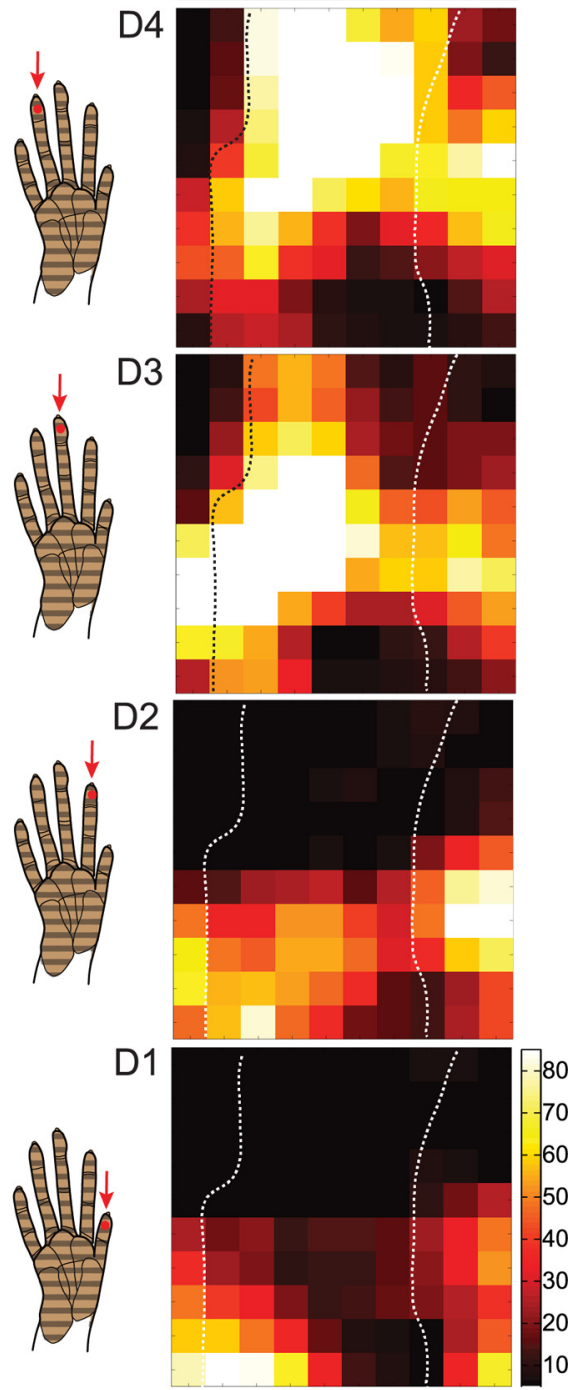

Figure 14. Responsiveness in partially deprived area 3b in Monkey OM-D.A,A transverse view of spinal cord showing the extent of lesion (black shading) reconstructed from a series of horizontally cut spinal cord sections. $\boldsymbol{B}$, Top. Schematic drawing shows an overview of an $0 \mathrm{M}$ brain. The array location is indicated by a box. Middle, Photomicrograph from a myelin-stained section cut parallel to pia surface through somatosensory areas $3 \mathrm{~b}$ and 1 shows the array location. Bottom, Schematic drawing shows a reconstructed somatotopic map from the array. $\boldsymbol{C}$, Heat maps of the peak firing rate responses across the array follow conventions of Figures 10-13 (color scale 5-85 spikes/s). Schematic drawings of $0 \mathrm{M}$ hands indicate the sites for tactile stimulation (red dots with arrows) on individual digits. Stripes on hands refer to partial deafferentation. Peak firing rate responses in territories of D3 and D4 were strong and extensive. Digits were represented in expected somatotopic order, but high firing rates during D3 or D4 stimulation were recorded from neurons expected to be found in neighboring digit territories. Dashed lines indicate rostral and caudal borders of area 3b. Conventions follow Figures 6 and 7.

key have been reported previously (Reed et al., 2008, 2010a,b, 2011, 2012).

To determine differential changes in submodalities after dorsal column lesion, we calculated ratios of neuronal responses to dorsal skin versus glabrous skin (Merzenich et al., 1984). Specifically, we tallied the number of electrodes in area $3 \mathrm{~b}$ that re- sponded to stimulation of the dorsal skin and/or hairs and divided by the total number of responding electrodes in area $3 \mathrm{~b}$ territory. The average proportions of electrodes responding to dorsal skin and/or hairs tended to be slightly higher in cases after DC lesion than in normal cases with no lesion ( $11 \%$ and $8 \%$, respectively; $p=0.222$; Wilcoxon-Mann-Whitney $\mathrm{U}=19$; total $n=10$ monkeys).

Quantitatively characterizing response fields: topography, discontinuity, and territory expansion

We calculated single-unit and multiunit responses to controlled mechanical stimulation on discrete hand locations ( $1 \mathrm{~mm}$ diameter contact surface). The purpose was to quantify neuronal responses to tactile stimulation and to detect response patterns over multiple digit representations simultaneously. Significant increases beyond baseline firing rates were visualized in a $10 \times 10$ grid, with a color map representing the peak firing rate magnitudes across neuron units sampled from the electrode array. Generating an image of cortical activity is similar to methods described by Rousche et al. (1999) and Normann et al. (2001) for Utah arrays and by Ghazanfar and Nicolelis (1997) for microwire arrays. To indicate approximate spatial locations of the electrodes, the firing rate map was overlaid on the topographical map of the area $3 \mathrm{~b}$ hand representation (obtained from RF mapping and reconstructions), as we have described previously (Reed et al., 2008, 2010b, 2011). (See Figures 10-14, heat maps, which are averages of neuron firing as a snapshot of brain activity.)

\section{Monkey OM-A}

Examples of neuronal responses to tactile stimulation of proximal D1, distal D2, and distal D3 from normal Monkey OM-A are shown in Figure $10 C$ for comparison with figures that follow for DC lesion cases. The averaged responses of peak firing rates to stimulation on a site on a single digit are somewhat widespread, but the activity tends to be focused within the corresponding digit territories determined by RF mapping. Stimulation of proximal digit 1 resulted in peak activity within the proximal D1 territory but was also associated with high firing rates in area 3 a digit and palm territory, possibly because of the proximity of the palm to the proximal digit stimulation.

\section{Monkey OM-M}

Typical examples of neuronal responses to tactile stimulation of distal D5, or distal D4, or proximal phalanx of D3 from the mon- 


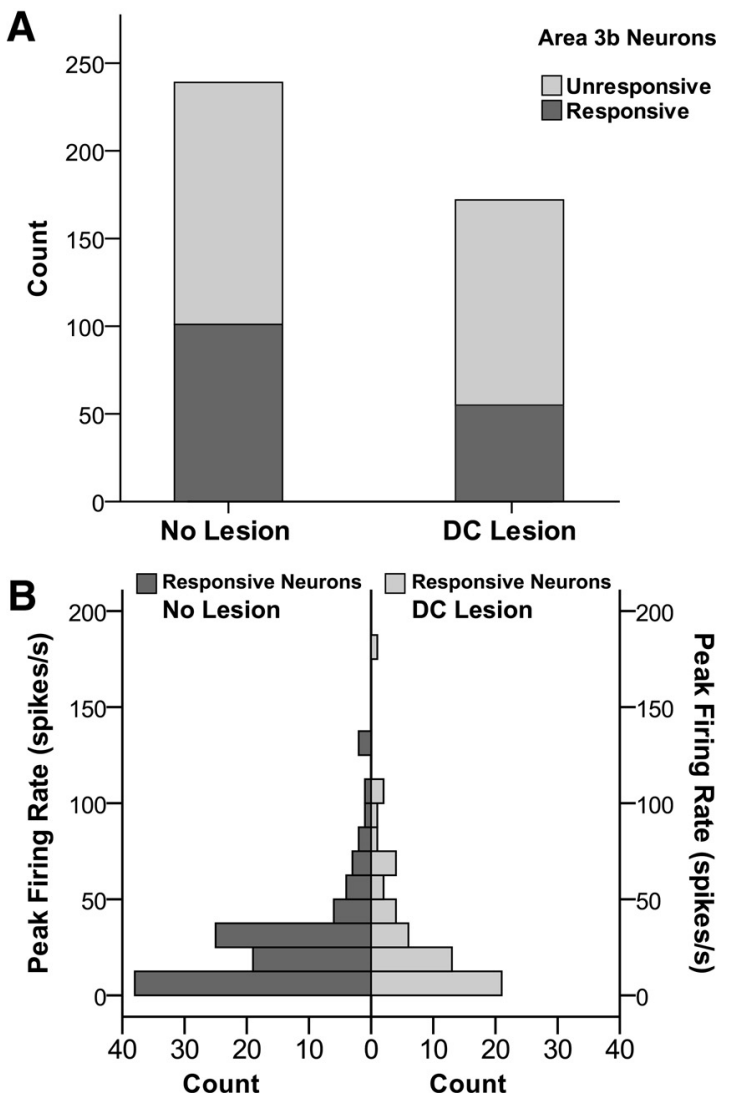

Figure 15. Counts of responsive neurons and peak firing rate distributions from wellisolated single neurons in area $3 \mathrm{~b} . \boldsymbol{A}, \boldsymbol{B}$, Data include 1 peak firing rate value per neuron per monkey (the maximum peak firing rate was selected from the recordings during tactile stimulation on different hand locations). $\boldsymbol{A}$, Counts divided into neurons that were unresponsive (light gray) or responsive (dark gray) to mechanical stimulation on tested hand locations in 5 normal monkeys with no lesion and 5 monkeys after DC lesion. Statistical differences were not detected between the distributions of responsive neurons in normal and lesion monkeys (101 of 239 and 55 of 172 , respectively). $\boldsymbol{B}$, Frequency distributions of peak firing responses to tactile stimulation on the hand were similar for neurons sampled from electrode array recordings in monkeys after DC lesions (light gray, 55 neurons) and in normal control monkeys with no lesion (dark gray, 101 neurons), with an overall median of 18.2 spikes/s.

key with the most extensive lesion are shown in Figure 11. Electrodes with neuronal responses to stimulation of digits 4 and 5 correspond to the same digit territories determined by RF mapping. Activations in area $3 \mathrm{~b}$ were weaker in response to distal D3 stimulation compared with that of D4 and D5, which is consistent with RF mapping. Responses were generally weak in this largely deprived area $3 \mathrm{~b}$ compared with cases with less extensive lesions.

\section{Monkey SM-G}

After incomplete C6 lesion, neuronal responses to tactile stimulation of D1-D5 activated expected cortical territories in lateral to medial sequence in area $3 \mathrm{~b}$ (Fig. 12B). When each digit was stimulated, a responding center with high peak firing rate could be found within appropriate digit territories determined by the RF mapping. Overall responsiveness in the hand representation of area $3 b$ was strong and widely spread when partially spared digits D1 and D2 were stimulated. In contrast, responses were much weaker and more restricted when deprived digits D3-D5 were stimulated (Fig. 12C). Notably, when spared digits D1 and D2 were stimulated, high magnitude responses were also found in largely deprived territories of digits 4 and 5 in a discontinuous, disorganized somatotopy (Fig. 12C). This abnormal response pattern was quantified and summarized across lesion cases and normal cases (see Relationships between axon sparing, behavioral recovery, and responsiveness of cortex within monkeys, including comparisons of response characteristics between normal and lesion monkeys). Expansion of the representations of spared territory into deprived cortex has been described (for review, see Jones, 2000; Kaas, 2000). However, we believe this is the first quantitative demonstration of disorganized activation in deprived area $3 \mathrm{~b}$ after incomplete DC lesion.

\section{Monkey OM-J}

After incomplete lesion ( $<50 \%$ of inputs spared), neuronal responses in partially deprived area $3 \mathrm{~b}$ demonstrated lateromedial shifts in response centers when stimulating D1-D5 (Fig. 13B). Activation centers corresponded to the expected digit territories determined from the RF map (Fig. 13C). However, overall responses were weak, somewhat restricted, and disorganized.

\section{Monkey OM-D}

After incomplete lesion (50\% of inputs spared), peak response centers to digit stimulation shifted lateromedially when stimulating D1-D5 and corresponded to the same digit territories in the RF map. Overall responsiveness was strong when stimulating digits D3 and D4, and the peak response centers were widespread (Fig. 14C). For example, when D3 was stimulated, the largest responses were not limited to D3 territory but also appeared in D1-D2 and D4-D5 territories. Similar effects were also observed from D4 stimulation (Fig. 14C).

\section{Relationships between axon sparing, behavioral recovery, and responsiveness of cortex within monkeys, including comparisons of response characteristics between normal and lesion monkeys \\ Single-unit responsiveness in normal and lesion monkeys}

We compared firing rates for well-isolated single units recorded in area $3 \mathrm{~b}$ in lesion and normal monkeys. We selected the maximum peak firing rate from all of the instances in which the same neuron was recorded over time so that each neuron would not be counted more than once. In normal monkeys $(n=5), 42 \%$ (101 of 239) of single units were responsive to mechanical stimulation on the hand locations tested and 58\% (138 of 239) were unresponsive. In lesion monkeys ( $n=5), 32 \%$ (55 of 172) of single units were responsive and $68 \%$ (117 of 172) were unresponsive (Fig. 15A). However, this difference in proportions of responsive neurons between normal and lesion monkeys was not significant ( $p=0.841$, Wilcoxon-Mann-Whitney $\mathrm{U}=11$ ). From the area $3 \mathrm{~b}$ neurons that responded to the hand locations tested, the distributions of peak firing rate responses were not significantly different ( $p=0.999$, Wilcoxon-Mann-Whitney $U=2777$ ) between lesion monkeys ( $n=55$ neurons) and normal monkeys ( $n=101$ neurons), as shown in Figure $15 B$. The median peak firing rate for responsive neurons was 18.2 spikes/s. Furthermore, we did not detect a correlation between the lesion extent (proportion of spared inputs) and firing rate magnitudes in area $3 \mathrm{~b}$ neurons in monkeys with and without DC lesions (Pearson's $r=$ $-0.033, p=0.679)$. Thus, after behavioral recovery from DC lesion, peak firing of single neurons in response to controlled stimulation on the hand was indistinguishable from the profile of neurons recorded in normal cortex.

Spatial patterns of single and multiunit responsiveness in normal and lesion monkeys

To examine patterns of activity across cortex covered by the array, we included multiunit and single-unit activity measures 
Table 2. Spread of activation and discontinuous response field tallies ${ }^{a}$

\begin{tabular}{|c|c|c|c|c|c|c|}
\hline Case & $\begin{array}{l}\text { Electrodes } \\
\text { (area 3b) }\end{array}$ & $\mathrm{RF} \geq 2$ sites & $\mathrm{RF}=1$ site & $\mathrm{RF}=0$ sites & $\begin{array}{l}\text { Discontinuous RF } \\
\text { ( } \geq 2 \text { sites) }\end{array}$ & $\begin{array}{l}\text { Continuous RF } \\
\text { ( } \geq 2 \text { sites) }\end{array}$ \\
\hline Deprived OM-M & 45 & $17(0.3778)$ & $15(0.3333)$ & $13(0.2889)$ & $3(0.1765)$ & $14(0.3111)$ \\
\hline Deprived SM-G & 62 & $25(0.4032)$ & $25(0.4032)$ & $12(0.1935)$ & $11(0.4400)$ & $14(0.2258)$ \\
\hline Deprived OM-D & 42 & $29(0.6905)$ & $5(0.1190)$ & $8(0.1905)$ & $9(0.3103)$ & $20(0.4762)$ \\
\hline Deprived 0M-J & 54 & $31(0.5741)$ & $8(0.1481)$ & $15(0.2778)$ & $8(0.2581)$ & $23(0.4340)$ \\
\hline Deprived SM-Ro & 23 & $16(0.6957)$ & $3(0.1304)$ & $4(0.1739)$ & $3(0.1875)$ & $13(0.5652)$ \\
\hline Deprived totals & 226 & $118(0.5221)$ & $56(0.2478)$ & $52(0.2301)$ & $34(0.2881)$ & $84(0.7119)$ \\
\hline Normal OM-L & 45 & $5(0.1111)$ & $16(0.3556)$ & $24(0.5333)$ & 0 & $5(0.1111)$ \\
\hline Normal OM-Ma & 38 & $1(0.0263)$ & $17(0.4474)$ & $20(0.5263)$ & 0 & $1(0.0263)$ \\
\hline Normal OM-Mo & 23 & $4(0.1739)$ & $5(0.2174)$ & $14(0.6087)$ & 0 & $4(0.1739)$ \\
\hline Normal OM-0 & 23 & $4(0.1739)$ & $12(0.5217)$ & $7(0.3043)$ & 0 & $4(0.1739)$ \\
\hline Normal OM-V & 68 & $43(0.6324)$ & $12(0.1765)$ & $13(0.1912)$ & $2(0.0465)$ & $41(0.6029)$ \\
\hline Normal totals & 197 & $57(0.2893)$ & $62(0.3147)$ & $78(0.3959)$ & $2(0.0351)$ & $55(0.9649)$ \\
\hline
\end{tabular}

${ }^{a}$ Based on quantified peak firing rates to determine response fields, the number of electrodes in which neurons responded to 2 or more sites on the hand, 1 site on the hand, or 0 sites tested on the hand were tallied for each monkey. The proportions of response types within each case are included. When neurons recorded from one electrode responded to 2 or more sites on the hand, the number of occurrences in which the sites were continuous or adjacent were tallied (continuous RF) compared with occurrences in which the sites were discontinuous or nonadjacent (discontinuous RF).

from each electrode in area $3 \mathrm{~b}$. We found more occasions when activity from one electrode responded with significant firing rate increases to more than one hand location in lesion cases than in normal cases, which is tallied for each case in Table 2. Occasionally, when activity from one electrode responded to more than one hand location, the hand sites were not adjacent or continuous, and we determined the proportion of multisite response fields that showed such discontinuity (discontinuous RF). Discontinuous RF proportions were greater in lesion cases than normal cases (median $=0.2581$ and 0 , respectively, Mann-Whitney $\mathrm{U}=15$, total $n=10$ monkeys, $p=0.008$ ). As an example at the single-unit level, Figure 16 shows peristimulus time histograms and raster plots from a neuron (unit 82b) from Monkey SM-G that did not respond to D2 stimulation but responded to stimulation on surrounding digits (D1, D3, D4). Such examples were rare; however, occurrences were more frequent in lesion cases than normal cases (Table 2). Although neurons in normal cases responded to more than one location on the hand, discontinuous RFs were only encountered in $3.5 \%$ of the sample (2 of 57).

To evaluate changes in digit territories after lesion, we calculated a ratio to compare a radial digit territory to an ulnar digit territory within each case. If the lesion spares some inputs, competition for territory after lesion could result in expansion of spared representations at the expense of deprived representations. If the ratio of radial-to-ulnar territory ( $\mathrm{R}-\mathrm{U}$ digit ratio) deviates from 1 , the territories are unequal. The $\mathrm{R}-\mathrm{U}$ digit ratio was close to 1 in normal and deprived monkeys ( median $=1.152$ and 0.8778 , respectively) and not significant (Mann-Whitney $\mathrm{U}=31$, total $n=10$ monkeys, $p=0.548$ ). In C4 lesion cases, the lesion did not spare some representations preferentially over others. As such, the R-U digit ratios for these cases were close to 1 , similar to normal cases. The C6 lesion case, Monkey SM-G, had an $\mathrm{R}-\mathrm{U}$ digit ratio of 1.75 , suggesting that the radial representation of the hand expanded at the expense of the ulnar hand territory in area $3 \mathrm{~b}$ (Fig. $7 \mathrm{~B}$ ). Because the lesion was at the C6 level, inputs from digits 1,2 , and possibly 3 were spared from the cut and appeared to expand in territory based on measures of firing activity. This R-U digit ratio for radial territory expansion corresponds to assessments from quantitative snapshots of single-unit and multiunit firing rate responses (Fig. 12).

Relationship between behavioral impairments, lesion characteristics, and response field properties of area $3 b$ We used correlation analyses to better characterize contributions to differences in cortical response properties between lesion and normal cases and to predict relationships between factors within lesion cases. Relationships between cortical reorganization, anatomical sparing, and behavioral impairment are shown in Figure 17. We expected strong correlations in relationships among all three factors. Within the 5 deprived cases, nonparametric correlations between measures for behavioral deficit rankings, proportion of spared inputs, firing rate response magnitudes, and cortical reorganization ratios ( $\mathrm{R}-\mathrm{U}$ digit ratios, discontinuous $\mathrm{RF}$ ratios) were not significantly different. However, as shown in Figure $5 C$, the behavioral deficit rankings tended to correlate well with the estimates of spared axons $(p=0.0598)$.

When the 5 normal and 5 deprived monkeys were examined together, we found that the discontinuous RF ratio was significantly correlated with the proportion of axons spared (Pearson's $r=-0.8162, p=0.004$ ) and behavioral deficit rank (Pearson's $r=-0.7708, p=0.009$ ), as shown in Figure 17A. The R-U digit territory ratio was not significantly correlated with other measures (Fig. 17B).

\section{Discussion}

After large, nearly complete lesions of the dorsal column pathway at a midcervical level of the spinal cord in adult monkeys, two remarkable recoveries occur over the ensuing 5-10 postlesion weeks. First, hand use in a food retrieval task is initially impaired but returns to normal over the first few weeks of testing (Qi et al., 2013). Second, as demonstrated here, the deprived hand representation in area $3 \mathrm{~b}$, which is initially unresponsive after almost all of the primary afferents from the hand that travel in the dorsal columns have been cut, undergoes extensive reactivation. We found that some aspects of recovery in reactivated cortex were similar across a range of lesion extents and resembled normal cortex in naive monkeys. Thus, a normal lateral to medial sequence of digit representations from 1 to 5 was largely restored in the hand region of somatosensory cortex, and neuron peak firing amplitudes returned to normal. On the other hand, the somatotopic and RF abnormalities that remained in reactivated cortex after behavioral recovery from DC lesions were closely related to lesion severity and behavioral impairments over the course of the recovery period.

\section{Area $3 \mathrm{~b}$ recovers response properties during behavioral recovery from dorsal column lesions of varying extents}

For all 5 monkeys with partial DC lesions, the somatotopy of the reactivated hand representation approximated that of normal 


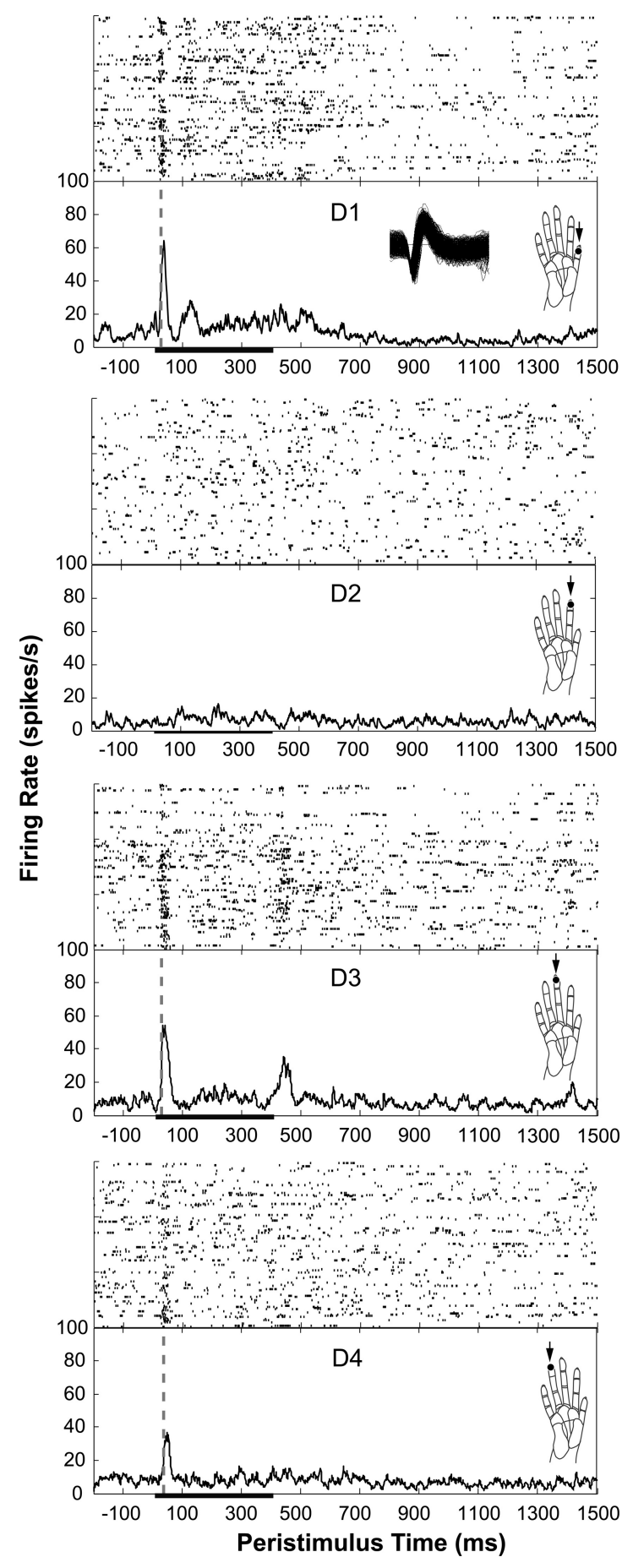

Figure 16. Series of peristimulus time histograms and rasters across stimulation locations show responses for unit 82b in Monkey SM-G, representing activation beyond one digit location with discontinuous activation patterns. Trace of waveforms from neuron $82 \mathrm{~b}$ is inset; trace window duration is $1.6 \mathrm{~ms}$. Stimulation was presented in blocks of 150 trials on digit locations indicated on drawings of monkey hands (D1-D4). Vertical dashed lines indicate the latency of the peak response. The duration for each stimulus was $400 \mathrm{~ms}$, indicated by the horizontal line.

monkeys (Merzenich et al., 1978; Sur et al., 1982), as previously reported (Jain et al., 1997, 2008; Qi et al., 2011; Chen et al., 2012). Unexpectedly, we found that reactivated neurons responded to tactile stimuli with peak amplitudes similar to those of normal monkeys studied with these recording methods (Reed et al., 2010a,b, 2011). Thus, after weeks of behavioral recovery, response magnitudes of neurons in area $3 \mathrm{~b}$ did not reflect the great reduction in inputs from the dorsal column pathway.
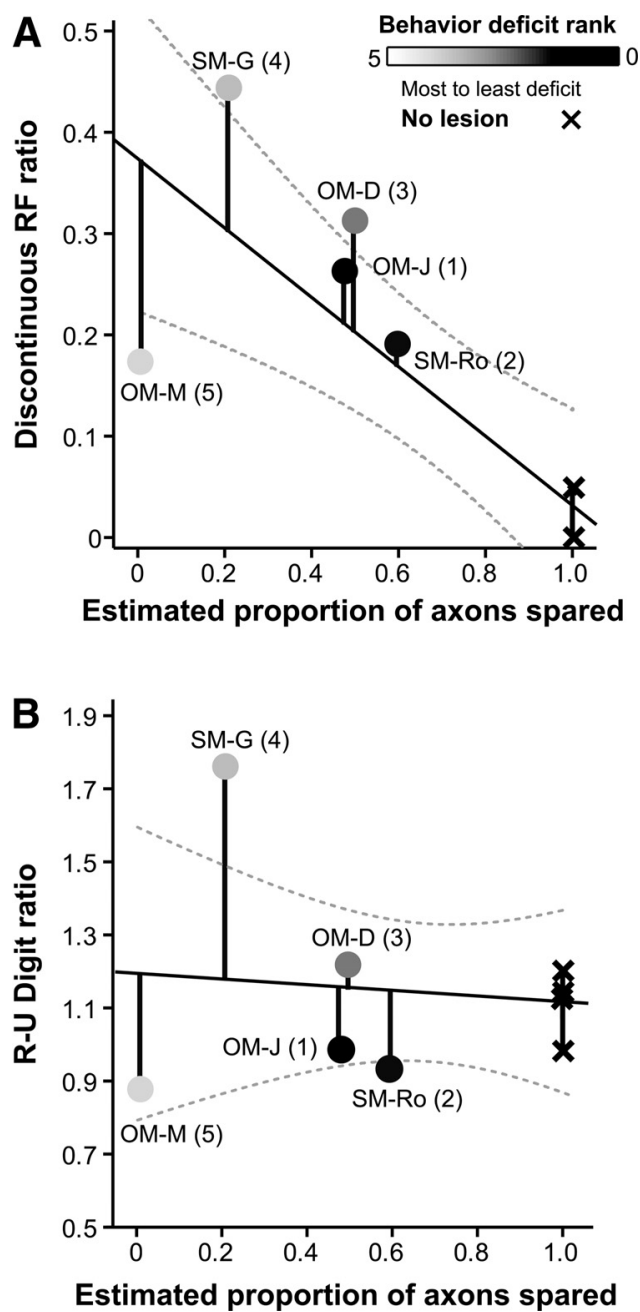

Figure 17. Relationships between lesion extent and cortical reactivation properties. $A$, Discontinuous $\mathrm{RF}$ ratio ( $y$-axis) is the ratio representing discontinuous response fields from the total multisite response fields. This measure is correlated with the estimated proportion of axons spared ( $x$-axis), as shown in a scatter plot for each monkey case. Normal cases are represented by a black X. Normal monkeys $(n=5)$ are plotted in the $x$-axis dimension as $100 \%$ spared, but some points overlap. The scale and conventions apply to both panels. Dashed lines indicate $95 \%$ confidence intervals based on the predicted mean linear regression fit line, shown as a solid line $\left(y=0.37-0.34 x, R^{2}=0.666\right)$. $B$, Relationship between the $R-U$ digit ratio ( $y$-axis) versus estimated proportion of axons spared ( $x$-axis) shown in a scatter plot for each monkey case. Dashed lines indicate $95 \%$ confidence intervals based on the predicted mean linear regression fit line, shown as a solid line ( $\left.y=1.19-0.08 x, R^{2}=0.014\right)$. Most normal and lesion cases had digit territory ratios near 1 , which is expected if no territories expand. Monkey SM-G (C6 lesion) showed evidence of radial digit territory expansion.

In addition, some features found predominately in reorganized cortex were also apparent in normal cortex, although to a more limited extent. Specifically, some neurons in normal cortex had multidigit RFs, and even discontinuous RFs. Small proportions of neurons in area $3 \mathrm{~b}$ of normal monkeys with multidigit response fields have been reported (Friedman et al., 2008; Reed et al., 2010a,b; Thakur et al., 2012). However, such reports have not noted the discontinuous territories of some of these RFs. Discontinuous RFs have been reported in cases with peripheral nerve damage and reinnervation errors (Merzenich et al., 1983a; Florence et al., 1994) and cases in which the face representation invades the cortical territory of the hand after injury or amputation (Pons et al., 1991; Moore et al., 2000; Jain et al., 2008). Here we show that discontinuities in response fields are common in cases 
of incomplete lesions that spare portions of sensory input from the hand and occasionally in monkeys without lesions.

Each monkey in the present study regained the ability to perform a reach-to-grasp task at prelesion levels over 5-10 weeks after lesion. Neurons across area $3 \mathrm{~b}$ were reactivated in a nearly normal somatotopic pattern, and neurons were driven by tactile stimulation with peak firing rates that did not differ from normal. These results suggested that cortical reorganization and behavioral recovery are highly correlated. More specifically, the somatosensory cortex of mature primates may reorganize in ways that appear to promote behavioral recovery after sensory loss, or the use of impaired hand may be a driving force to promote cortical reorganization, or both.

\section{Abnormal cortical properties after behavioral recovery from dorsal column lesions relate to lesion site and extent}

Previous studies established that the mature somatosensory system adapts in response to peripheral nerve section or digit amputations (Merzenich et al., 1983a,b; Calford and Tweedale, 1988; Garraghty and Kaas, 1991; Florence et al., 1998), dorsal root transections (Pons et al., 1991; Darian-Smith and Brown, 2000; Darian-Smith and Ciferri, 2005), and dorsal column injuries (Jain et al., 1997, 2008; Qi et al., 2011). However, to our knowledge, this is the first report of quantified measures of behavioral impairment, lesion severity, and cortical response properties within the same monkeys, which then allows us to characterize the relationships of these factors after DC injury. We found that increased proportions of neurons with discontinuities in response fields strongly correlated with decreased proportions of axons spared by the lesion. Additionally, we found that the proportions of neurons with discontinuous response fields correlated strongly with behavioral impairments ranked over the entire recovery period rather than the endpoint at which all cases achieved success. However, the case with the most extensive lesion (Monkey OM-M with $<1 \%$ axon sparing) did not have the largest proportion of discontinuities. Instead, cases with lesions that spared $\sim \geq 20 \%$ of the DC axons had greater proportions of discontinuous response fields. Although further study is needed, the differences in cortical response properties in Monkey OM-M compared with the other cases suggest that, when the lesion is nearly complete, the mechanisms of behavioral recovery differ.

We propose that there are two modes of cortical reactivation underlying our results. One mode, based on the growth and potentiation of preserved DC afferents, likely dominates when significant amounts of axons are preserved (Jain et al., 2000; for review, see Kaas et al., 2008). Axon arbor atrophy or withdrawal in the affected dorsal column pathway, as demonstrated after midcervical DC lesions in monkeys, likely promotes the growth of spared axons into deactivated regions of brainstem and cortex (Graziano and Jones, 2009), leading to abnormal somatotopy. A second mode involves the potentiation of subthreshold secondorder spinal cord inputs to the cuneate nucleus (for review, see Abraira and Ginty, 2013) and the spinothalamic pathway. When inputs are substantially reduced by extensive lesions, secondary pathway potentiation would produce a largely normal somatotopy, as axon growth to new targets is limited without interventions (for review, see Kaas et al., 2008). In addition, the limited rebalancing of somatosensory networks that can be revealed immediately after sensory loss (e.g., Calford, 2002; for review, see Aguilar et al., 2010) may play a small role in the functional state of the cortex we examined at the later endpoint at 5-10 weeks after lesion.

\section{Significance and future directions for understanding mediators of recovery after dorsal column lesions}

Our present results are important in that they allow us to compare, quantitatively, features of reactivated cortex in relation to measures of lesion completeness and behavioral recovery within and across cases. Simultaneous recordings from ensembles of neurons in somatosensory cortex reveal response patterns that may be missed by single-electrode mapping studies. Here, array recordings showed how neurons in different parts of the cortical hand representation were simultaneously active when a discrete location on a single digit was stimulated, even in monkeys with extensive dorsal column lesions. The fixed electrode position in the 100 electrode array eliminates variations in the spacing of recording sites that might confound comparisons across lesioned and normal cases, and the use of a controlled electromechanical stimulator eliminates variability in stimulation parameters that would limit comparisons of response magnitudes across cases. Our quantified measures of response territories in cortex largely agree with the qualitative assessments obtained in previous studies involving DC lesions in monkeys (e.g., Jain et al., 1997, 2008; Qi et al., 2011). These previous studies established that most of the deprived cortex reactivates even after extensive dorsal column lesions, but we were surprised to find in the present study that peak response amplitudes to controlled stimulation returned to normal. In addition, our current results uniquely relate quantified measures of reactivated cortex to the lesion extent and behavioral recovery in individual monkeys.

Finally, we emphasize that the amount of reactivation that occurs after our most complete lesion is difficult to explain by the presence of preserved DC afferents alone. Using quantified measures of response territories and cortical organization, somatotopy and neuron properties in area $3 \mathrm{~b}$ were surprisingly normal, even after an extensive DC lesion in Monkey OM-M. The mechanisms that result in the reactivation of cortex and in a largely normal somatotopic organization after such extensive lesions are unclear. In previous reports, we have stressed the major role of preserved dorsal column afferents, but this explanation now seems insufficient given our quantification of lesions and the observation that behavioral recovery still occurs after extensive lesions. Although answers are not yet known, it seems likely that second-order neurons in the spinal cord, which are activated by peripheral nerve cutaneous afferents and project to the dorsal column nuclei, and possibly spinothalamic inputs that remain intact after dorsal column lesions play a prominent role in recovery. Although extensive efforts have been underway to promote recoveries from spinal cord injury (Onifer et al., 2011; for review, see Bradbury and Carter, 2011; Filli and Schwab, 2012; GarcíaAlías and Fawcett, 2012; Yoon and Tuszynski, 2012; Houle and Côté, 2013), our research on spontaneous recovery after incomplete lesions suggests that both the enhancement of the effectiveness of secondary pathways and the promotion of the survival and growth of even a few primary afferents will have great impact on functional recovery.

\section{References}

Abraira VE, Ginty DD (2013) The sensory neurons of touch. Neuron 79: 618-639. CrossRef Medline

Aguilar J, Humanes-Valera D, Alonso-Calviño E, Yague JG, Moxon KA, Oliviero A, Foffani G (2010) Spinal cord injury immediately changes the state of the brain. J Neurosci 30:7528-7537. CrossRef Medline

Bradbury EJ, Carter LM (2011) Manipulating the glial scar: chondroitinase $\mathrm{ABC}$ as a therapy for spinal cord injury. Brain Res Bull 84:306-316. CrossRef Medline

Bruce K, Grofova I (1992) Notes on a light and electron microscopic 
double-labeling method combining anterograde tracing with Phaseolus vulgaris leucoagglutinin and retrograde tracing with cholera toxin subunit B. J Neurosci Methods 45:23-33. CrossRef Medline

Calford MB (2002) Dynamic representational plasticity in sensory cortex. Neuroscience 111:709-738. CrossRef Medline

Calford MB, Tweedale R (1988) Immediate and chronic changes in responses of somatosensory cortex in adult flying-fox after digit amputation. Nature 332:446-448. CrossRef Medline

Chen LM, Qi HX, Kaas JH (2012) Dynamic reorganization of digit representations in somatosensory cortex of nonhuman primates after spinal cord injury. J Neurosci 32:14649-14663. CrossRef Medline

Darian-Smith C, Brown S (2000) Functional changes at periphery and cortex following dorsal root lesions in adult monkeys. Nat Neurosci 3:476481. CrossRef Medline

Darian-Smith C, Ciferri MM (2005) Loss and recovery of voluntary hand movements in the macaque following a cervical dorsal rhizotomy. J Comp Neurol 491:27-45. CrossRef Medline

Filli L, Schwab ME (2012) The rocky road to translation in spinal cord repair. Ann Neurol 72:491-501. CrossRef Medline

Florence SL, Wall JT, Kaas JH (1989) Somatotopic organization of inputs from the hand to the spinal gray and cuneate nucleus of monkeys with observations on the cuneate nucleus of humans. J Comp Neurol 286:4870. Medline

Florence SL, Wall JT, Kaas JH (1991) Central projections from the skin of the hand in squirrel monkeys. J Comp Neurol 311:563-578. CrossRef Medline

Florence SL, Garraghty PE, Wall JT, Kaas JH (1994) Sensory afferent projections and area $3 \mathrm{~b}$ somatotopy following median nerve cut and repair in macaque monkeys. Cereb Cortex 4:391-407. CrossRef Medline

Florence SL, Taub HB, Kaas JH (1998) Large-scale sprouting of cortical connections after peripheral injury in adult macaque monkeys. Science 282: 1117-1121. CrossRef Medline

Friedman RM, Chen LM, Roe AW (2008) Responses of areas 3b and 1 in anesthetized squirrel monkeys to single- and dual-site stimulation of the digits. J Neurophysiol 100:3185-3196. CrossRef Medline

Gallyas F (1979) Silver staining of myelin by means of physical development. Neurol Res 1:203-209. Medline

García-Alías G, Fawcett JW (2012) Training and anti-CSPG combination therapy for spinal cord injury. Exp Neurol 235:26-32. CrossRef Medline

Garraghty PE, Kaas JH (1991) Large-scale functional reorganization in adult monkey cortex after peripheral nerve injury. Proc Natl Acad Sci U S A 88:6976-6980. CrossRef Medline

Gharbawie OA, Stepniewska I, Kaas JH (2011) Cortical connections of functional zones in posterior parietal cortex and frontal cortex motor regions in New World monkeys. Cereb Cortex 21:1981-2002. CrossRef Medline

Ghazanfar AA, Nicolelis MA (1997) Nonlinear processing of tactile information in the thalamocortical loop. J Neurophysiol 78:506-510. Medline

Graziano A, Jones EG (2009) Early withdrawal of axons from higher centers in response to peripheral somatosensory denervation. J Neurosci 29: 3738-3748. CrossRef Medline

Houle JD, Côté MP (2013) Axon regeneration and exercise-dependent plasticity after spinal cord injury. Ann N Y Acad Sci 1279:154-163. CrossRef Medline

Jain N, Catania KC, Kaas JH (1997) Deactivation and reactivation of somatosensory cortex after dorsal spinal cord injury. Nature 386:495-498. CrossRef Medline

Jain N, Florence SL, Kaas JH (1998) Reorganization of somatosensory cortex after nerve and spinal cord injury. News Physiol Sci 13:143-149. Medline

Jain N, Florence SL, Qi HX, Kaas JH (2000) Growth of new brainstem connections in adult monkeys with massive sensory loss. Proc Natl Acad Sci U S A 97:5546-5550. CrossRef Medline

Jain N, Qi HX, Collins CE, Kaas JH (2008) Large-scale reorganization in the somatosensory cortex and thalamus after sensory loss in macaque monkeys. J Neurosci 28:11042-11060. CrossRef Medline

Jones EG (2000) Cortical and subcortical contributions to activitydependent plasticity in primate somatosensory cortex. Annu Rev Neurosci 23:1-37. CrossRef Medline

Kaas JH (2000) The reorganization of somatosensory and motor cortex after peripheral nerve or spinal cord injury in primates. Prog Brain Res 128:173-179. CrossRef Medline
Kaas JH, Merzenich MM, Killackey HP (1983) The reorganization of somatosensory cortex following peripheral nerve damage in adult and developing mammals. Annu Rev Neurosci 6:325-356. CrossRef Medline

Kaas JH, Qi HX, Burish MJ, Gharbawie OA, Onifer SM, Massey JM (2008) Cortical and subcortical plasticity in the brains of humans, primates, and rats after damage to sensory afferents in the dorsal columns of the spinal cord. Exp Neurol 209:407-416. CrossRef Medline

Merzenich MM, Kaas JH, Sur M, Lin CS (1978) Double representation of the body surface within cytoarchitectonic areas $3 \mathrm{~b}$ and 1 in "SI" in the owl monkey (Aotus trivirgatus). J Comp Neurol 181:41-73. CrossRef Medline

Merzenich MM, Kaas JH, Wall J, Nelson RJ, Sur M, Felleman D (1983a) Topographic reorganization of somatosensory cortical areas $3 \mathrm{~b}$ and 1 in adult monkeys following restricted deafferentation. Neuroscience 8:3355. CrossRef Medline

Merzenich MM, Kaas JH, Wall JT, Sur M, Nelson RJ, Felleman DJ (1983b) Progression of change following median nerve section in the cortical representation of the hand in areas $3 \mathrm{~b}$ and 1 in adult owl and squirrel monkeys. Neuroscience 10:639-665. CrossRef Medline

Merzenich MM, Nelson RJ, Stryker MP, Cynader MS, Schoppmann A, Zook JM (1984) Somatosensory cortical map changes following digit amputation in adult monkeys. J Comp Neurol 224:591-605. Medline

Moore CI, Stern CE, Dunbar C, Kostyk SK, Gehi A, Corkin S (2000) Referred phantom sensations and cortical reorganization after spinal cord injury in humans. Proc Natl Acad Sci U S A 97:14703-14708. CrossRef Medline

Nelson RJ, Sur M, Felleman DJ, Kaas JH (1980) Representations of the body surface in postcentral parietal cortex of Macaca fascicularis. J Comp Neurol 192:611-643. CrossRef Medline

Nicolelis MA, Dimitrov D, Carmena JM, Crist R, Lehew G, Kralik JD, Wise SP (2003) Chronic, multisite, multielectrode recordings in macaque monkeys. Proc Natl Acad Sci U S A 100:11041-11046. CrossRef Medline

Normann RA, Warren DJ, Ammermuller J, Fernandez E, Guillory S (2001) High-resolution spatio-temporal mapping of visual pathways using multi-electrode arrays. Vision Res 41:1261-1275. CrossRef Medline

Onifer SM, Smith GM, Fouad K (2011) Plasticity after spinal cord injury: relevance to recovery and approaches to facilitate it. Neurotherapeutics 8:283-293. CrossRef Medline

Pons TP, Garraghty PE, Ommaya AK, Kaas JH, Taub E, Mishkin M (1991) Massive cortical reorganization after sensory deafferentation in adult macaques. Science 252:1857-1860. CrossRef Medline

Qi HX, Kaas JH (2006) Organization of primary afferent projections to the gracile nucleus of the dorsal column system of primates. J Comp Neurol 499:183-217. CrossRef Medline

Qi HX, Chen LM, Kaas JH (2011) Reorganization of somatosensory cortical areas $3 \mathrm{~b}$ and 1 after unilateral section of dorsal columns of the spinal cord in squirrel monkeys. J Neurosci 31:13662-13675. CrossRef Medline

Qi HX, Gharbawie OA, Wynne KW, Kaas JH (2013) Impairment and recovery of hand use after unilateral section of the dorsal columns of the spinal cord in squirrel monkeys. Behav Brain Res 252:363-376. CrossRef Medline

Reed JL, Pouget P, Qi HX, Zhou Z, Bernard MR, Burish MJ, Haitas J, Bonds $\mathrm{AB}, \mathrm{Kaas} \mathrm{JH}$ (2008) Widespread spatial integration in primary somatosensory cortex. Proc Natl Acad Sci U S A 105:10233-10237. CrossRef Medline

Reed JL, Qi HX, Pouget P, Burish MJ, Bonds AB, Kaas JH (2010a) Modular processing in the hand representation of primate primary somatosensory cortex coexists with widespread activation. J Neurophysiol 104:31363145. CrossRef Medline

Reed JL, Qi HX, Zhou Z, Bernard MR, Burish MJ, Bonds AB, Kaas JH (2010b) Response properties of neurons in primary somatosensory cortex of owl monkeys reflect widespread spatiotemporal integration. J Neurophysiol 103:2139-2157. CrossRef Medline

Reed JL, Qi HX, Kaas JH (2011) Spatiotemporal properties of neuron response suppression in owl monkey primary somatosensory cortex when stimuli are presented to both hands. J Neurosci 31:3589-3601. CrossRef Medline

Reed JL, Pouget P, Qi HX, Zhou Z, Bernard MR, Burish MJ, Kaas JH (2012) Effects of spatiotemporal stimulus properties on spike timing correlations in owl monkey primary somatosensory cortex. J Neurophysiol 108:33533369. CrossRef Medline

Rousche PJ, Petersen RS, Battiston S, Giannotta S, Diamond ME (1999) 
Examination of the spatial and temporal distribution of sensory cortical activity using a 100-electrode array. J Neurosci Methods 90:57-66. CrossRef Medline

Samonds JM, Allison JD, Brown HA, Bonds AB (2003) Cooperation between area 17 neuron pairs enhances fine discrimination of orientation. J Neurosci 23:2416-2425. Medline

Shoham S, Fellows MR, Normann RA (2003) Robust, automatic spike sorting using mixtures of multivariate $t$-distributions. J Neurosci Methods 127:111-122. CrossRef Medline

Sur M, Nelson RJ, Kaas JH (1982) Representations of the body surface in cortical areas $3 \mathrm{~b}$ and 1 of squirrel monkeys: comparisons with other primates. J Comp Neurol 211:177-192. CrossRef Medline

Thakur PH, Fitzgerald PJ, Hsiao SS (2012) Second-order receptive fields reveal multidigit interactions in area $3 \mathrm{~b}$ of the macaque monkey. J Neurophysiol 108:243-262. CrossRef Medline

Tommerdahl M, Whitsel BL, Vierck CJ Jr, Favorov O, Juliano S, Cooper B,
Metz C Nakhle B (1996) Effects of spinal dorsal column transection on the response of monkey anterior parietal cortex to repetitive skin stimulation. Cereb Cortex 6:131-155. CrossRef Medline

Wang Z, Qi HX, Kaas JH, Roe AW, Chen LM (2013) Functional signature of recovering cortex: dissociation of local field potentials and spiking activity in somatosensory cortices of spinal cord injured monkeys. Exp Neurol 249:132-143. CrossRef Medline

Wong-Riley M (1979) Changes in the visual system of monocularly sutured or enucleated cats demonstrable with cytochrome oxidase histochemistry. Brain Res 171:11-28. CrossRef Medline

Yoon C, Tuszynski MH (2012) Frontiers of spinal cord and spine repair: experimental approaches for repair of spinal cord injury. Adv Exp Med Biol 760:1-15. Medline

Zhou Z, Bernard MR, Bonds AB (2008) Deconstruction of spatial integrity in visual stimulus detected by modulation of synchronized activity in cat visual cortex. J Neurosci 28:3759-3768. CrossRef Medline 